\title{
THE FIRST Hi-GAL OBSERVATIONS OF THE OUTER GALAXY: A LOOK AT STAR FORMATION IN THE THIRD GALACTIC QUADRANT IN THE LONGITUDE RANGE $216^{\circ} .5 \lesssim \ell \lesssim 225^{\circ} .5$
}

\author{
D. Elia ${ }^{1}$, S. Molinari ${ }^{1}$, Y. Fukui ${ }^{2}$, E. Schisano ${ }^{1,3}$, L. Olmi $^{4,5}$, M. Veneziani $^{3}$, T. Hayakawa ${ }^{2}$, M. Pestalozzi ${ }^{1}$,

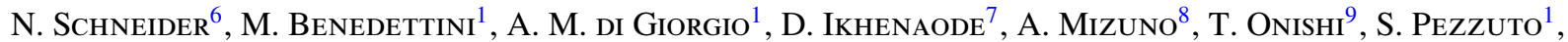 \\ L. Piazzo ${ }^{6}$, D. Polychroni ${ }^{10}$, K. L. J. RYGL ${ }^{1}$, H. YAMAMOTO ${ }^{2}$, And Y. Maruccia ${ }^{11}$ \\ ${ }^{1}$ Istituto di Astrofisica e Planetologia Spaziali-INAF, Via Fosso del Cavaliere 100, I-00133 Roma, Italy; davide.elia@iaps.inaf.it \\ ${ }^{2}$ Department of Physics, Nagoya University, Furo-cho, Chikusa-ku, Nagoya 464-8602, Japan \\ ${ }^{3}$ Infrared Processing and Analysis Center, California Institute of Technology, Pasadena, CA 91125, USA \\ ${ }^{4}$ Osservatorio Astrofisico di Arcetri-INAF, Largo E. Fermi 5, I-50125 Firenze, Italy \\ ${ }^{5}$ University of Puerto Rico, Rio Piedras Campus, Physics Department, Box 23343, UPR Station, San Juan, PR, USA \\ ${ }^{6}$ IRFU/SAp CEA/DSM, Laboratoire AIM CNRS, Universit Paris Diderot, F-91191 Gif-sur-Yvette, France \\ ${ }^{7}$ DIET-Dipartimento di Ingegneria dell'Informazione, Elettronica e Telecomunicazioni, Università di Roma La Sapienza, via Eudossina 18, I-00184 Roma, Italy \\ ${ }^{8}$ Solar-Terrestrial Environment Laboratory, Nagoya University, Furo-cho, Chikusa-ku, Nagoya 464-8601, Japan \\ ${ }^{9}$ Department of Physical Science, Osaka Prefecture University, Sakai, Osaka 599-8531, Japan \\ ${ }^{10}$ Department of Astrophysics, Astronomy and Mechanics, Faculty of Physics, University of Athens, Panepistimiopolis, 15784 Zografos, Athens, Greece \\ ${ }^{11}$ Dipartimento di Matematica e Fisica, Università del Salento, CP 193, I-73100 Lecce, Italy \\ Received 2013 January 22; accepted 2013 April 25; published 2013 July 5
}

\begin{abstract}
We present the first Herschel PACS and SPIRE photometric observations in a portion of the outer Galaxy (216.5 $\lesssim \ell \lesssim 225.5$ and $-2^{\circ} \lesssim b \lesssim 0^{\circ}$ ) as a part of the Hi-GAL survey. The maps between 70 and $500 \mu \mathrm{m}$, the derived column density and temperature maps, and the compact source catalog are presented. NANTEN CO $(1-0)$ line observations are used to derive cloud kinematics and distances so that we can estimate distance-dependent physical parameters of the compact sources (cores and clumps) having a reliable spectral energy distribution that we separate into 255 proto-stellar and 688 starless sources. Both typologies are found in association with all the distance components observed in the field, up to $\sim 5.8 \mathrm{kpc}$, testifying to the presence of star formation beyond the Perseus arm at these longitudes. Selecting the starless gravitationally bound sources, we identify 590 pre-stellar candidates. Several sources of both proto- and pre-stellar nature are found to exceed the minimum requirement for being compatible with massive star formation based on the mass-radius relation. For the pre-stellar sources belonging to the Local arm $(d \lesssim 1.5 \mathrm{kpc})$ we study the mass function whose high-mass end shows a power law $N(\log M) \propto M^{-1.0 \pm 0.2}$. Finally, we use a luminosity versus mass diagram to infer the evolutionary status of the sources, finding that most of the proto-stellar sources are in the early accretion phase (with some cases compatible with a Class I stage), while for pre-stellar sources, in general, accretion has not yet started.
\end{abstract}

Key words: Galaxy: structure - infrared: ISM - ISM: clouds - ISM: molecules - stars: formation

Online-only material: color figures, extended figure, machine-readable tables

\section{INTRODUCTION}

Looking at star formation across the Milky Way, a large variety of different conditions are encountered, in many cases leading to different observed modalities. One of the most relevant differences is found in the amount of star formation activity between the inner and the outer Galaxy. The smaller content of star formation in the outer Galaxy is mainly related to its lower density of atomic and molecular hydrogen compared with that of the inner Galaxy (Wouterloot et al. 1990).

Nevertheless, the outer Galaxy offers the chance to study the interstellar clouds and possible ongoing processes of star formation with a lower degree of confusion, also close to the Galactic plane. Furthermore, reconstructing the structure of the velocity field from line observations is easier in this case, compared to the inner Galaxy, thanks to the lack of distance ambiguities at any observed radial velocity.

In this paper, we present the first photometric study of a portion of the outer Galaxy surveyed by the Herschel Space Observatory (Pilbratt et al. 2010) as part of the Hi-GAL key project (Molinari et al. 2010b), selecting a range of longitudes $\left(216.5 \lesssim \ell \lesssim 225^{\circ} .5\right)$ in the third Galactic quadrant (TGQ), also covered by a $\mathrm{CO}(1-0)$ line map obtained with the NANTEN radio telescope. This combination of FIR/submillimeter dust continuum maps at unprecedented resolution and sensitivity and the information about kinematics provided by the line observations allow us to investigate the first stages of star formation in this portion of the Milky Way by estimating and discussing in a statistical sense the physical properties of the compact sources (cores and clumps) detected in the Herschel maps. With this analysis we aim at shedding more light on the star formation in the TGQ, to which a relatively little amount of literature has been devoted so far.

The first extensive surveys of the TGQ were carried out by May et al. $(1988,1993)$ at angular resolutions of 0.5 and 8 ' 8 , respectively. They confirmed the warping of the disk at these longitudes (Burton \& te Lintel Hekkert 1986) and the presence of weak emission from a molecular cloud located at Galactocentric distances larger than $R=12 \mathrm{kpc}$. The latter survey was then drawn by May et al. (1997) to derive the properties of the clouds located beyond $2 \mathrm{kpc}$ from the Sun. A relevant finding of this analysis is that no grand design spiral pattern is seen in the distribution of the clouds on the Galactic disk. The warp of the Galaxy in the TGQ has also been observed by Wouterloot et al. (1990) and Dobashi et al. (2005) through the distribution of the IRAS sources and of the visual extinction maps derived from the Digitized Sky Survey I, respectively.

To assess the spiral structure of the Galaxy in the TGQ, other techniques have also been adopted. Carraro et al. (2005) and Moitinho et al. (2006) have studied the distribution of 
young star clusters, tracing the Local, the Perseus, and the Norma-Cygnus (Outer) arms. This latter is not detected at these longitudes by Nakanishi \& Sofue (2006) based on the Dame et al. (2001) CO survey (which is taken, for most of the TGQ, from that of May et al. 1993). Instead, the Moitinho et al. (2006) results were confirmed by Vázquez et al. (2008), who attribute to the outer arm a grand design feature extending from $\ell=190^{\circ}$ to $255^{\circ}$ (reaching heliocentric distances $d=$ 6 and $12 \mathrm{kpc}$ at the two extreme longitudes, respectively), expanding the sample of clusters of Moitinho et al. (2006) and combining this information with kinematics extracted from new CO observations (still unpublished at present).

Focusing on the vicinity of the region analyzed in this work, the most important star-forming region is CMa R1, dominated by the presence of the CMa OB1 association (Ruprecht 1966) located at $d=1150 \mathrm{pc}$, i.e., in the Local arm, and with an age of $3 \times 10^{6}$ yr (Clariá 1974) and of the Sh2-296 (S296 in the simplified notation we adopt hereafter) H II region (Sharpless 1959). The population of young stars in CMa R1 has been studied by Gregorio-Hetem et al. (2009) based on X-ray data, and its typical age has been estimated as older than $10^{7} \mathrm{yr}$. However, only a very small fraction of the investigated protostars lie in the area surveyed in this paper.

This area was fully covered, instead, by the survey of molecular clouds in the Monoceros and Canis Major regions carried out with the NANTEN telescope in the ${ }^{13} \mathrm{CO}(1-0)$ line (Kim et al. 2004), spanning an area of $560 \mathrm{deg}^{2}$. The two most massive giant molecular clouds found are the aforementioned CMa OB1 and G220.8-1.7 (Maddalena et al. 1986), the latter one located at $d=1050 \mathrm{pc}$. These authors also studied the relation between the gas emission morphology and the star formation signatures that have been observed in this region, as several IRAS young stellar object (YSO) candidates and HII regions (Sharpless 1959; Blitz et al. 1982). The low level of extinction $\left(A_{V}<3\right)$ found by Dobashi et al. (2005) in the entire region, however, seems to exclude the possibility of encountering massive star formation (MSF; cf., e.g., Krumholz \& McKee 2008). The western part of our survey corresponds, instead, to the northeastern corner of G216-2.5 (or Maddalena's cloud, $d=2200 \mathrm{pc}$ ), mapped in both $\mathrm{CO}(1-0)$ and ${ }^{13} \mathrm{CO}(1-0)$ by Lee et al. (1994). These authors claim the non-star-forming nature for this cloud, partially controverted by more recent Spitzer observations of Megeath et al. (2009), while recognizing the star-forming character of a nearby filamentary cloud associated with the $\mathrm{S} 287 \mathrm{H}$ II region, fully covered by our observations.

In this framework, the new Hi-GAL observations in the crucial range $70-500 \mu \mathrm{m}$ will permit us to obtain a more exhaustive description of the star-forming activities or capabilities in this region. In particular, both the early stages of the proto-stellar core evolution and the properties of their pre-stellar progenitors can be studied, being in both cases the peak of the cold dust emission lying in this range of wavelengths (e.g., Molinari et al. 2010a; Elia et al. 2010).

Thanks to NANTEN CO(1-0) data, we are also able to estimate kinematic distances for those sources needed to derive masses and luminosities and then to depict a possible evolutionary scenario for the region. This allows us to carry out a complete study of cold dust and gas properties at unprecedented resolution and sensitivity in a significant portion of the outer Galaxy.

This analysis is structured and presented as follows. In Section 2 the details about Herschel and NANTEN observations are reported together with our data reduction strategy. In Section 3, and also in Appendix A, the obtained maps are presented and used to provide an overall description of the column density and temperature distributions in the surveyed area. Starting with Section 4, the discussion revolves around the compact sources that are extracted from the Hi-GAL maps and from the ancillary $\mathrm{CO}(1-0)$ observations through cloud decomposition techniques. In Section 5, the main properties of the Hi-GAL compact sources are derived from photometric data by means of a modified blackbody fit and then discussed in the framework of the differences between pre- and protostellar sources. In Section 6, in particular, these quantities are exploited to situate the Hi-GAL sources into an evolutionary picture. Finally, in Section 7 a summary of the obtained results is reported.

\section{OBSERVATIONS AND DATA REDUCTION}

\subsection{Hi-GAL Observations}

The Herschel Infrared Galactic plane survey (Hi-GAL; Molinari et al. 2010b) is a Herschel open-time key project that initially aimed at covering the inner Galaxy $\left(|\ell|<60^{\circ},|b|<\right.$ $1^{\circ}$ ), and was subsequently extended to the entire Galactic disk.

In this paper we publish the first four "tiles" observed in the TGQ as part of this survey, spanning the range of Galactic longitudes $216.5 \lesssim \ell \lesssim 225.5$ and the range of latitudes $-2^{\circ} \lesssim b \lesssim 0^{\circ}$, because in this portion of the Galactic plane the Hi-GAL observations do not straddle the latitude $b=0^{\circ}$, but follow the warped midplane.

The $2.3 \times 2.3$ fields presented in this paper were observed between 2011 May 5 and 9. As in the case of the whole Hi-GAL survey, the maps were obtained with the Herschel fast scan parallel mode, i.e., using simultaneously PACS at 70 and $160 \mu \mathrm{m}$ (Griffin et al. 2010) and SPIRE at 250, 350, and $500 \mu \mathrm{m}$ (Poglitsch et al. 2010) at a scan speed of $60^{\prime \prime} \mathrm{s}^{-1}$, and performing two scan maps of each field, one with nominal coverage and the other with orthogonal coverage, in order to increase data redundancy. Due to the $21^{\prime}$ separation of the PACS and SPIRE focal plane footprints on the sky, the areas covered by these two instruments are slightly different, namely, shifted with respect to each other in terms of this angular quantity. The diffraction limits at the five bands are 5".0, 11".4, 17".6, 23".9, and $35^{\prime \prime}$. 1, respectively. However, due to the PACS on-board data averaging and the use of the fast speed mode a degradation of the PACS point-spread function (PSF) is produced along the scan direction, up to $12^{\prime \prime} .2$ and $15^{\prime \prime} .7$ at 70 and $160 \mu \mathrm{m}$, respectively (PACS manual, available from the ESA Herschel Science Centre). The Hi-GAL observation naming convention consists of naming the tiles using the approximated value of the central longitude, so in the following we will designate these four tiles as $\ell 217,{ }^{12} \ell 220, \ell 222$, and $\ell 224$. Hereafter, we will call the entire region composed by these four tiles and analyzed in this paper $\ell 217-224$.

The data reduction strategy followed the Hi-GAL pipeline described in Traficante et al. (2011). Briefly, we used scripts written in the Herschel interactive processing environment (HIPE; Ott 2010) to reduce archival data to Level 1. The obtained time-ordered data (TODs) of each bolometer were then processed further by means of dedicated IDL routines to remove spurious instrumental effects and glitches due to

\footnotetext{
12 In this case, the value $\ell=218^{\circ}$ would be closer to the center of the map, but we use the conventional name already reported in the Herschel Data Archive.
} 


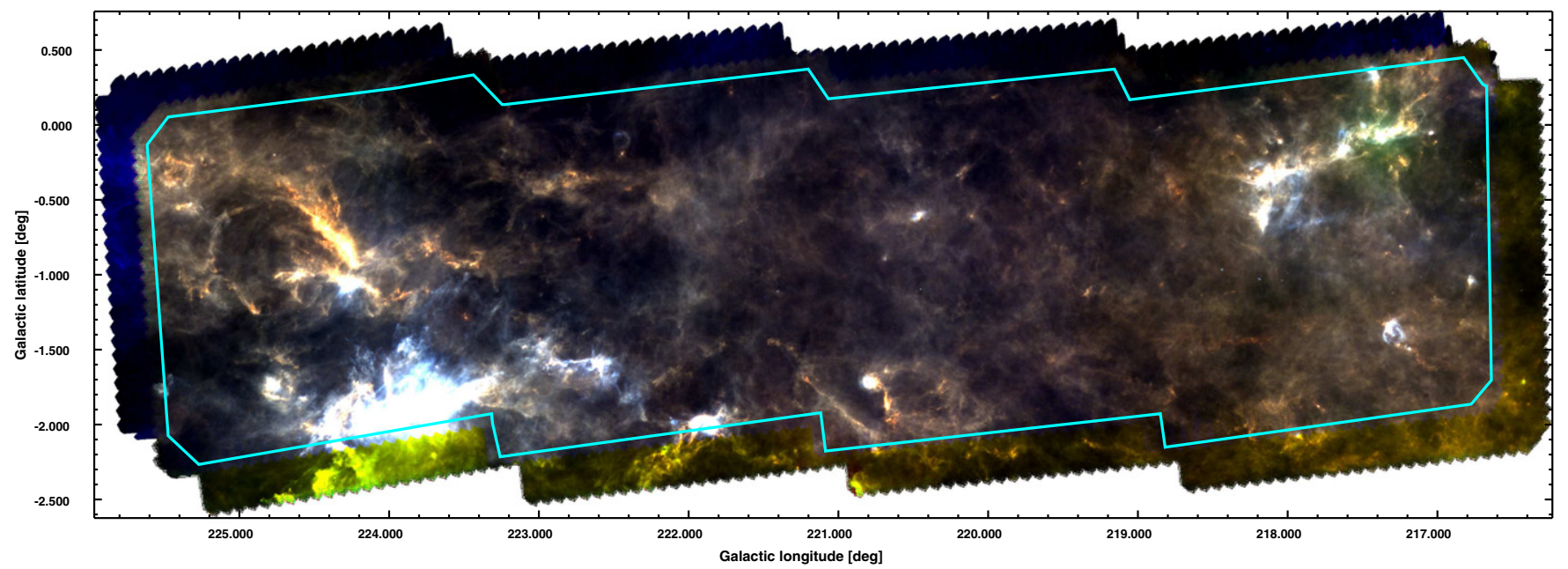

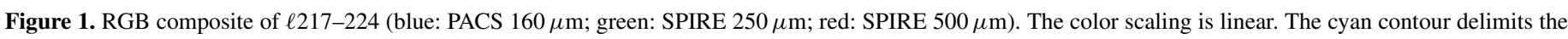
common science area covered by both PACS and SPIRE.

(A color version of this figure is available in the online journal.)

cosmic rays, and maps were obtained using the FORTRAN code ROMAGAL based on the Generalized Least Square (GLS; e.g., Tegmark 1997) approach, which was able to reduce the influence of the $1 / f$ noise in the TODs. Since the GLS technique is known to introduce artifacts in the maps, namely crosses and stripes corresponding to the brightest sources, a weighted postprocessing of the GLS maps (WGLS; Piazzo et al. 2012) has been applied to finally obtain images in which artifacts are removed or heavily attenuated.

The pixel sizes of the maps are 3 .'2, 4".5, 6.'0, 8.'0, and $11^{\prime \prime} .5$ at $70,160,250,350$, and $500 \mu \mathrm{m}$, respectively. The astrometry of the maps has been checked comparing the positions of several compact sources in the $70 \mu \mathrm{m}$ maps with those of their possible WISE (Wright et al. 2010) counterparts at $22 \mu \mathrm{m}$, and the calculated rigid offset applied for correction. Finally, the absolute calibration has been definitively performed by applying a linear transform of the maps adopting the coefficients determined by comparing Herschel with IRAS and Planck, following Bernard et al. (2010).

All the single tile images obtained at the five Hi-GAL wavelengths are reported in Figure 18 in Appendix A. In Figure 1, instead, we show a composite RGB image of the region, assembled combining the mosaics of the four tiles at 160 , 250 , and $500 \mu \mathrm{m}$. The $70 \mu \mathrm{m}$ observations have not been used because the diffuse emission they contain appears dominated by the noise and only the brightest regions are well recognizable. The mosaics have been produced in the same way as the single tiles, combining the TODs of the four tiles to obtain an overall map. The common science area, i.e., the area covered by PACS and SPIRE, highlighted with a cyan contour in Figure 1, amounts to $\sim 20.5 \mathrm{deg}^{2}$.

\subsection{NANTEN CO(1-0) Observations}

The NANTEN $\operatorname{CO}(J=1-0)$ Galactic Plane Survey data set (Mizuno \& Fukui 2004) was used in the present analysis. At the time of the observations, carried out in a period from 1996 to 2003, the 4 m NANTEN millimeter-wave telescope of Nagoya University was installed at Las Campanas Observatory in Chile. The half-power beamwidth was 2'.6. The data were gathered using the position switching mode. The pointing accuracy was better than $20^{\prime \prime}$. The observations covered a region of $\sim 23^{\circ}$ (the coordinates of the bottom-left and top-right pixels are $[\ell, b]=$ $[225.60,-2.53]$ and $[\ell, b]=\left[216.53,0^{\circ}\right]$, respectively), and the observed grid consists of $136 \times 38$ points located every $4^{\prime}$.

The spectrometer used was acousto-optical with 2048 channels providing a frequency resolution of $250 \mathrm{kHz}$. The spectral intensities were calibrated by employing the standard roomtemperature chopper wheel technique (Kutner \& Ulich 1981). An absolute intensity calibration was achieved by taking the absolute peak antenna temperature, $T_{\mathrm{R}}^{*}$, of Orion KL (R.A. = $5^{\mathrm{h}} 32^{\mathrm{m}} 47^{\mathrm{s}} .0$, decl. $=-5^{\circ} 24^{\prime} 21^{\prime \prime}$ for the equinox of 1950.0$)$ to be $65 \mathrm{~K}$. The rms noise fluctuations were typically $0.2 \mathrm{~K}$ with about $5 \mathrm{~s}$ integration for an on-position. A first-order degree polynomial was subtracted from the line spectra to account for instrumental baseline effects. Finally, antenna temperatures were divided by the main beam efficiency factor $\eta_{\mathrm{mb}}=0.89$ (Ogawa et al. 1990) to obtain main beam temperatures.

The integrated intensity maps obtained from these line observations are presented in Section 3.1.

\section{THE OVERALL DISTRIBUTION OF GAS AND DUST}

$$
\text { 3.1. }{ }^{12} \mathrm{CO}(1-0) \text { Line }
$$

A first look at the spectra in the reduced $\mathrm{CO}(1-0)$ data cube reveals that the structure of the velocity field is relatively clear due to the small number of spectral components detected and to the infrequent presence of more than one along the same line of sight.

A global view of the velocity field is provided in Figure 2 in which we show the sum of all the spectra composing the cube, where at least three main components are found, peaking at the 17,28 , and $40 \mathrm{~km} \mathrm{~s}^{-1}$ velocity channels, respectively. A secondary peak of the second component also appears around $33 \mathrm{~km} \mathrm{~s}^{-1}$. Finally, a further weak component, the most redshifted, is present around $54 \mathrm{~km} \mathrm{~s}^{-1}$. These indications can be exploited to determine opportune velocity ranges $\left[v_{0}, v_{1}\right]$ to build meaningful channel maps rather than blindly choosing equally spaced ranges of channels.

The maps have been obtained by integrating the line emission (main beam temperature) in those lines of sight where a feature with a signal-to-noise ratio $(\mathrm{S} / \mathrm{N})$ better than 3 has been found (the average baseline rms noise of the data cube 
Table 1

Overall Properties of the ${ }^{12} \mathrm{CO}(1-0)$ Emission in $\ell 217-224$

\begin{tabular}{|c|c|c|c|c|c|c|c|c|}
\hline Component & $\begin{array}{c}v_{\text {peak }} \\
\left(\mathrm{km} \mathrm{s}^{-1}\right)\end{array}$ & $\begin{array}{c}v_{0} \\
\left(\mathrm{~km} \mathrm{~s}^{-1}\right)\end{array}$ & $\begin{array}{c}v_{1} \\
\left(\mathrm{~km} \mathrm{~s}^{-1}\right)\end{array}$ & $\begin{array}{l}\text { Ref. Longitude } \\
\left({ }^{\circ}\right)\end{array}$ & $\begin{array}{l}\text { Distance } \\
(\mathrm{kpc})\end{array}$ & $\%$ of Total Emission & $\begin{array}{c}X \\
\times 10^{-20}\left(\mathrm{~cm}^{-2} \mathrm{~K}^{-1} \mathrm{~km}^{-1} \mathrm{~s}\right)\end{array}$ & $\begin{array}{c}\text { Mass } \\
\left(10^{5} M_{\odot}\right)\end{array}$ \\
\hline I & 17 & 29.5 & 51.5 & 223 & 1.1 & 69.2 & 3.2 & 1.1 \\
\hline II & 28 & 51.5 & 66.5 & 218 & 2.2 & 26.2 & 3.3 & 1.9 \\
\hline III & 40 & 66.5 & 75.5 & 222 & 3.3 & 4.3 & 3.5 & 0.7 \\
\hline IV & 54 & 75.5 & 94.5 & 217 & 5.8 & 0.4 & 4.0 & 0.2 \\
\hline
\end{tabular}

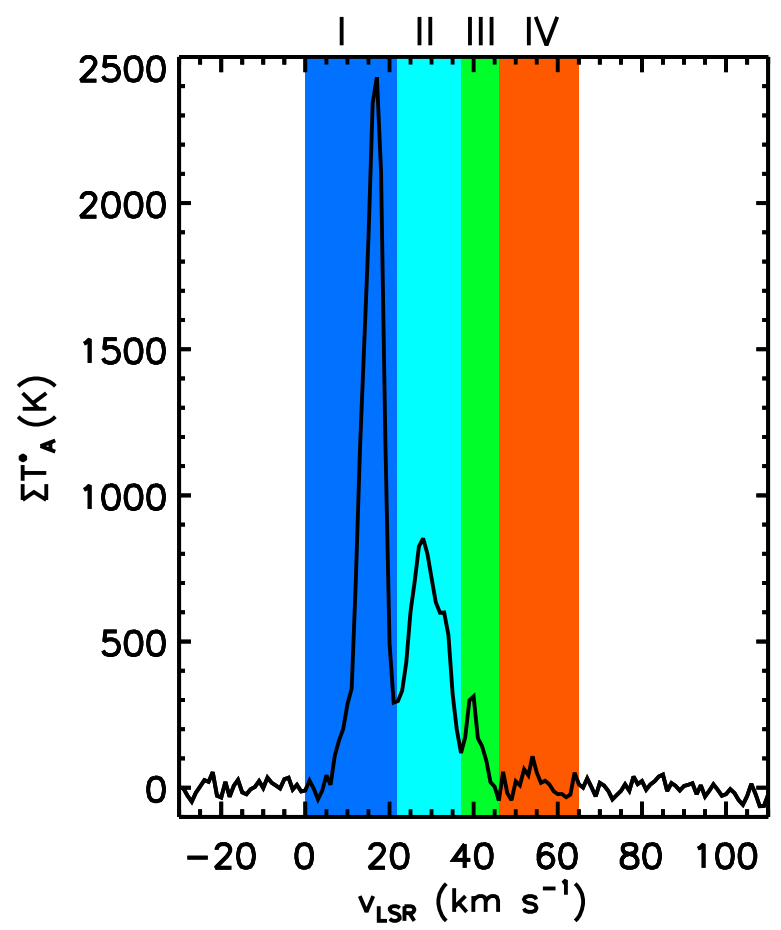

Figure 2. Sum of all the ${ }^{12} \mathrm{CO}(1-0)$ spectra. The four velocity ranges delimited by $-0.5,20.5,36.5,44.5$, and $65.5 \mathrm{~km} \mathrm{~s}^{-1}$ are labeled with Roman numerals I, II, III, and IV and highlighted with a blue, cyan, green, and red background, respectively.

(A color version of this figure is available in the online journal.)

is $\left.\sigma_{\mathrm{rms}}=0.23 \mathrm{~K}\right)$ :

$$
I_{\mathrm{CO}}=\int_{v_{0}}^{v_{1}} T_{\mathrm{mb}}^{*} d v .
$$

In Figure 3, we show both the map of the ${ }^{12} \mathrm{CO}(1-0)$ intensity integrated over the whole range from -0.5 to $60.5 \mathrm{~km} \mathrm{~K} \mathrm{~s}^{-1}$ (panel (a)) and the set of the maps corresponding to the ranges highlighted in Figures 2(b)-(e).

The first and second velocity components (sorted from the bluest to the reddest) are evidently predominant in the total budget of the emission. To quantify this, in Table 1, the contribution of each component to the integrated intensity map in panel (a) is quoted together with the average distance calculated at a characteristic longitude for each component. A gross estimate of the masses of these four components can be obtained given the information on the global distance. First, the local column density map is derived directly from the maps in Figure 3 through the empirical relation

$$
N\left(\mathrm{H}_{2}\right)_{i, j}=X \times I_{\mathrm{CO} i, j},
$$

where the conversion coefficient value adopted in this case is calculated as a function of the Galactocentric radius $R$ through
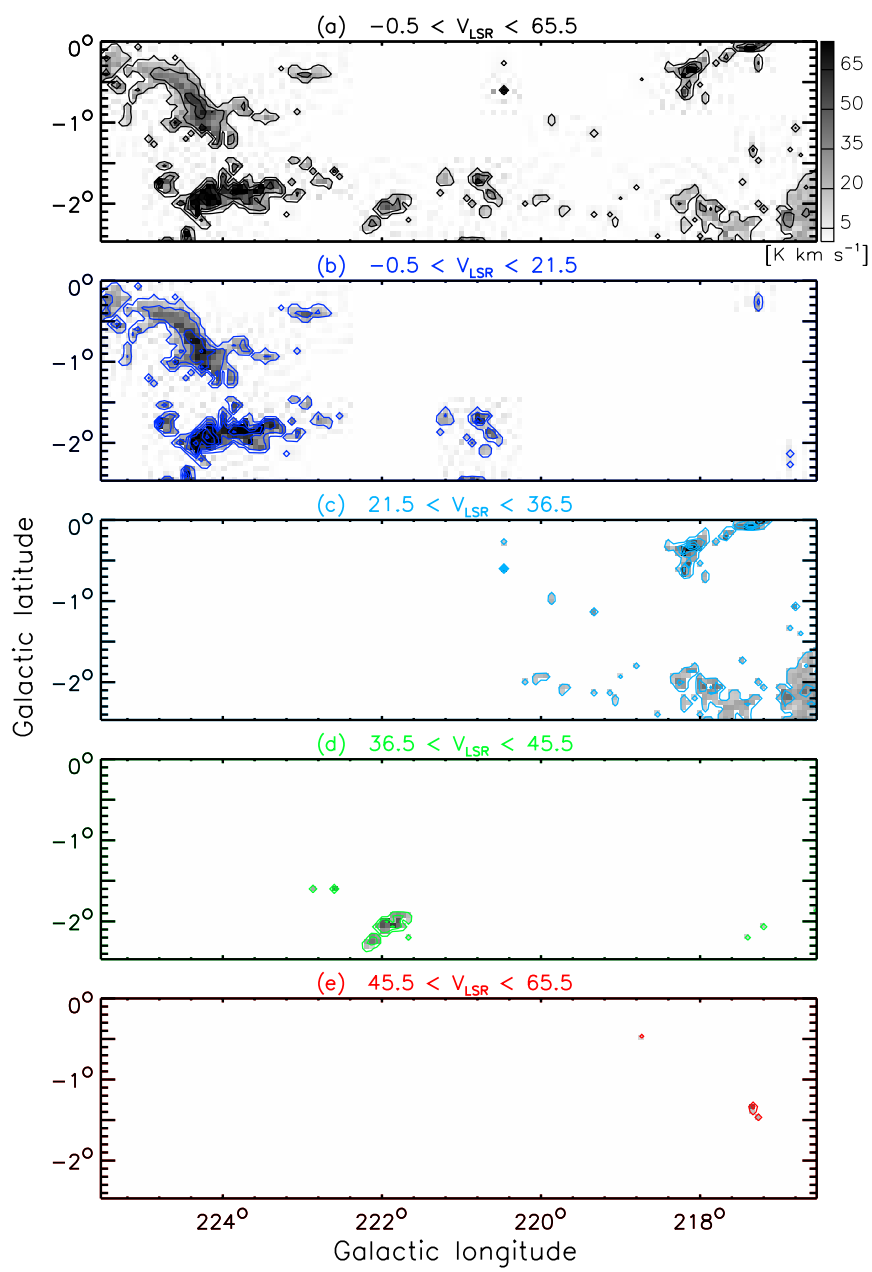

Figure 3. $\mathrm{CO}(1-0)$ integrated intensity maps of the $\ell 217-224$ region, in units of $\mathrm{K} \mathrm{km} \mathrm{s}^{-1}$. The correction for the $\eta_{\mathrm{mb}}$ factor is taken into account. In panel (a), the map obtained by integrating over the entire considered velocity range is shown, while panels (b)-(e) contain the intensity integrated over the ranges specified in the title of each panel. For all panels, contours start from $5 \mathrm{~K} \mathrm{~km} \mathrm{~s}^{-1}$ and are in steps of $15 \mathrm{~K} \mathrm{~km} \mathrm{~s}^{-1}$.

(A color version of this figure is available in the online journal.)

the relation of Nakanishi \& Sofue (2006):

$$
X\left[\mathrm{~cm}^{-2} \mathrm{~K}^{-1} \mathrm{~km}^{-1} \mathrm{~s}\right]=1.4 \times 10^{20} \exp (R / 11 \mathrm{kpc}) .
$$

Then, for each component the mass is obtained by integrating the column density over the area occupied in the sky: $M=$ $\sum_{i, j} N\left(\mathrm{H}_{2}\right)_{i, j} d^{2} \Delta \vartheta^{2} \mu m_{\mathrm{H}}$, where $\Delta \vartheta$ indicates the pixel scale of the $\mathrm{CO}(1-0)$ map (in radians).

Nevertheless, the value of the conversion coefficient $X$ is a matter of debate and strong variations of the obtained mass values are expected if another estimate of $X$ is adopted. For instance, Nakanishi \& Sofue (2006) make a twofold analysis using both the approach described by Equation (2) and the 


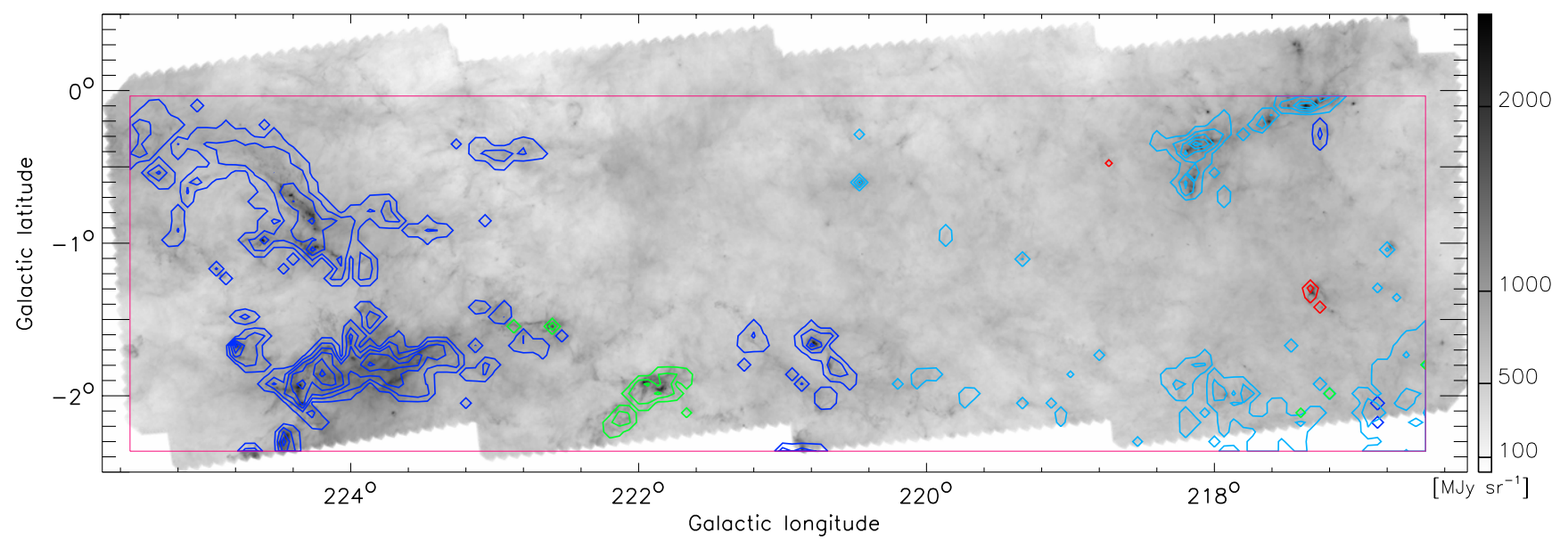

Figure 4. Structure of the velocity field in $\ell 217-224$. The contours of Figures 3(b)-(e) are overplotted, with the same color convention, on the SPIRE $250 \mu \mathrm{m}$ mosaic, while the magenta box represents the boundaries of the area surveyed in the $\mathrm{CO}(1-0)$ line. The dynamics of the SPIRE image has been compressed between 20 and $2500 \mathrm{MJy} \mathrm{sr}^{-1}$, and the scale is logarithmic.

(A color version of this figure is available in the online journal.)

constant value $X=1.8 \times 10^{20} \mathrm{~cm}^{-2} \mathrm{~K}^{-1} \mathrm{~km}^{-1} \mathrm{~s}$ of Dame et al. (2001). In our case, this latter value would lead to an underestimate of the masses in Table 1 of a factor from 1.8 to 2.2, depending on the distance.

The overall distribution of the column density derived from these maps is presented in Section 3.2, while the correspondence with Hi-GAL maps and with previous $\mathrm{CO}(1-0)$ surveys is discussed in Section 3.3. Here we just highlight the low degree of complexity of the velocity field, as it emerges from these line observations. In Figure 4, the $\mathrm{CO}(1-0)$ contours of Figures 3(b)-(e) overlap the SPIRE $250 \mu \mathrm{m}$ mosaic. The degree of overlap among components appears relatively low: the eastern part of the region is dominated by the first (bluest) component, and the western one is dominated by the second component, whereas the third and fourth components are basically associated with a few small and bright features of the SPIRE map.

\subsection{Herschel Temperature and Column Density Maps}

Both Herschel and NANTEN observations provide the possibility of deriving maps of the column density in the region. Possible differences between the resulting maps are expected due to the assumptions made on the considered component of the interstellar medium (ISM). Therefore, any comparison requires care and must be accompanied with some caveats.

We obtained the maps of column density and temperature for $\ell 217-224$ from the mosaics of the four Hi-GAL tiles, using the wavelengths from 160 to $500 \mu \mathrm{m}$, the emission at $70 \mu \mathrm{m}$ being generally contaminated by warmer dust components such as circumstellar matter and $\mathrm{H}$ II regions (cf. Schneider et al. 2012). This implies, of course, that these maps basically represent the properties of the cold component of the dust (large grains). From the practical point of view, considering only the common science area, all the maps have been rebinned onto the grid of the $500 \mu \mathrm{m}$ one, and a pixel-to-pixel modified blackbody fit has been performed, according to the usual expression

$$
F_{v}=N\left(\mathrm{H}_{2}\right) \mu m_{\mathrm{H}} \Delta \vartheta_{500}^{2} k_{0}\left(\frac{v}{v_{0}}\right)^{\beta} B_{v}(T),
$$

where $F_{v}$ represents the spectral energy distribution (SED) of the pixel, $\Delta \vartheta_{500}$ is the map pixel scale at $500 \mu \mathrm{m}$ in $\mathrm{sr}$, $k_{0}=0.1 \mathrm{~cm}^{2} \mathrm{~g}^{-1}$ at $\nu_{0}=1200 \mathrm{GHz}\left(\lambda_{0}=250 \mu \mathrm{m}\right)$ already accounting for a gas-to-dust ratio of 100 (Hildebrand 1983), $m_{\mathrm{H}}$ is the atomic hydrogen mass, and $\mu$ is the mean molecular weight, assumed to be equal to 2.8 to take into account a relative helium abundance of $25 \%$ in mass. We imposed $\beta=2$ in order to reduce the number of free parameters in the fit (see also Sadavoy et al. 2012).

The obtained column density and temperature maps are shown in Figures 5 and 6. Combining the information contained in these maps and in that of Figure 4 with the global impression provided by Figure 1, we are now able to provide an overall description of the $\ell 217-224$ region.

The easternmost tile, namely $\ell 224$, is by far the richest in bright features and, apparently, in star formation activity. All these regions belong to the CMa OB1 giant molecular cloud, located at $d=1150$ pc (Kim et al. 2004, and references therein), and are encircled by the H II region S296 (Sharpless 1959), as illustrated in Figure 5.

A large cavity centered at about $[\ell, b]=[224.8,-0.9]$ is well recognizable, with a filamentary ridge that turns out to be particularly massive and cold $(11 \mathrm{~K} \lesssim T \lesssim 13 \mathrm{~K})$ in the western part. Another bright filament aligned with the east-west direction seems to connect, in perspective, the center with the ridge of the cavity. This corresponds to the massive $\left(M=1.2 \times 10^{3} M_{\odot}\right)$ cloud 4 of the paper of Kim et al. (2004, [KKY2004]4 according to the SIMBAD nomenclature). The southern part of $\ell 224$ is dominated by another extended, bright and warmer region corresponding to S292 and [KKY2004]3; this latter is the most massive cloud $\left(M=1.9 \times 10^{3} M_{\odot}\right)$ of those identified by Kim et al. (2004) in the area considered in this paper. Cold filaments coexist with warm $(T<20 \mathrm{~K})$ diffuse emission or star-forming sites such as S292. The young cluster NGC 2335, located to the north of this region $([\ell, b]=$ $[223.62,-1.26])$ and with distance and age estimated as $1.79 \mathrm{pc}$ and 79 Myr, respectively (Moitinho et al. 2006), is not detected at any of the Herschel wavelengths.

In the $\ell 222$ tile another small cloud is found, [KKY2004]1, still belonging to CMa OB1. In the southern part, the BFS64 H II region (Blitz et al. 1982) is associated with the velocity component III. The emission in $\ell 220$, again, is mostly associated with the velocity component I. Two H II regions, BFS61 and BFS62, represent the signature of recent high-mass star formation activity, albeit much less dramatic than in $\ell 224$. 


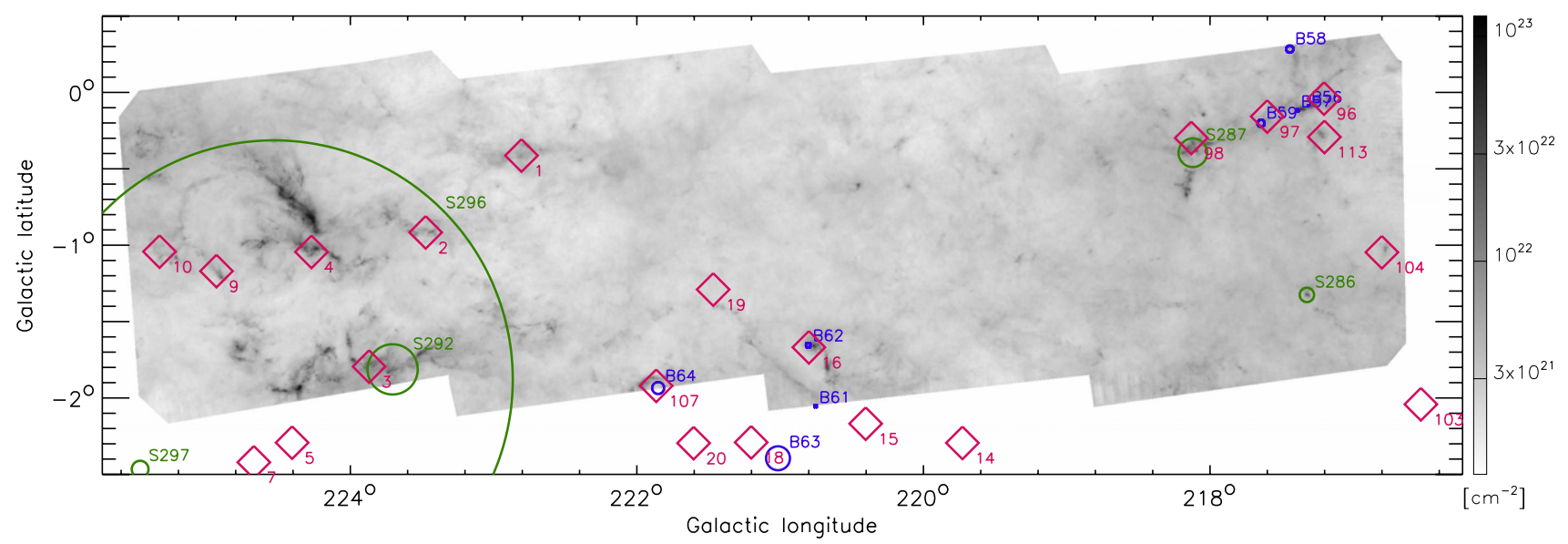

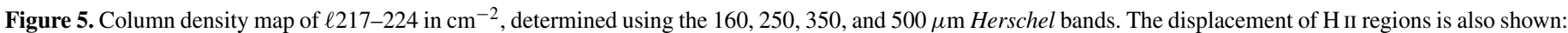
green circles are from Sharpless (1959) and blue circles are from Blitz et al. (1982). The object names, labeled at the top right of each region have been shortened to avoid confusion in the figure (only those of BFS56 and BFS57 are partially overlapped in the northwest part of the map). Finally, the molecular clouds of Kim et al. (2004) are overlapped as magenta diamonds, simply labeled with their running number at the bottom right.

(A color version of this figure is available in the online journal.)

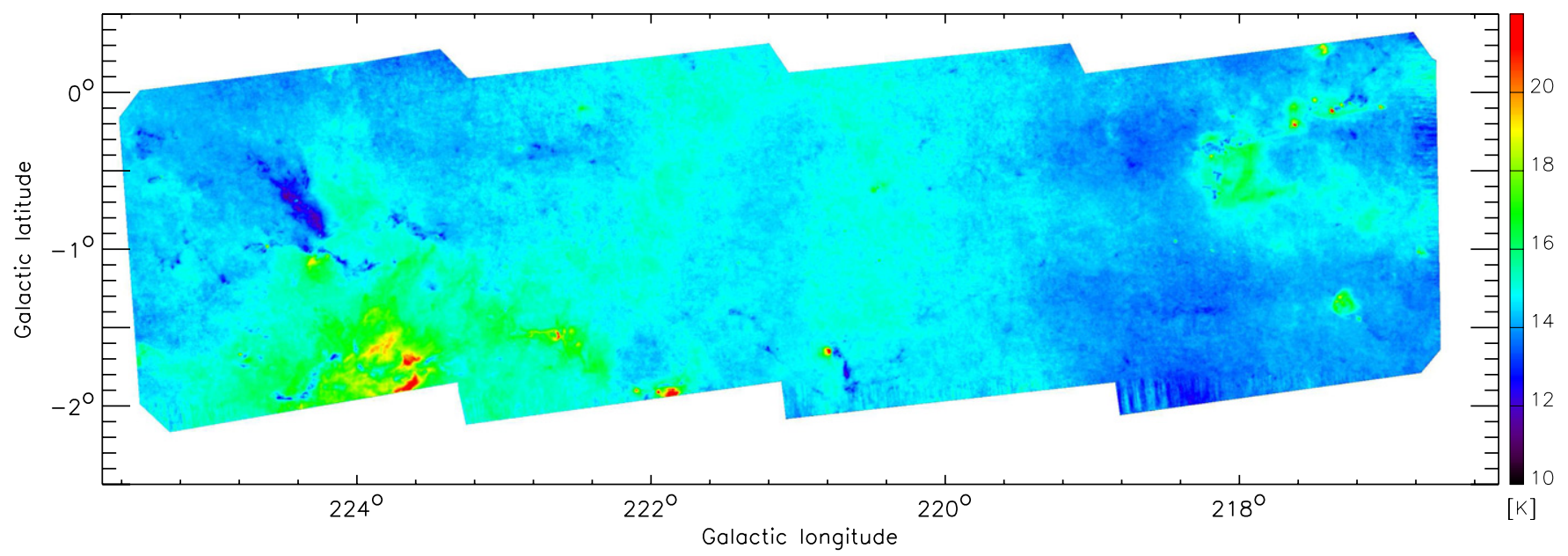

Figure 6. Temperature map of $\ell 217-224$, determined using the 160, 250, 350, and $500 \mu \mathrm{m}$ Herschel bands.

(A color version of this figure is available in the online journal.)

In $\ell 217$ a filamentary cloud already mentioned in Section 1 is prominent in the northern part. It belongs to the velocity component II and is associated with the S287 H II region and other minor ones, and with four molecular clouds of Kim et al. (2004). The angular size of the whole filament is approximately 1.6 , corresponding to an extent of $59 \mathrm{pc}$ at a distance of $d=2.1 \mathrm{kpc}$. It is considered an active site of star formation by Lee et al. (1994), who justify its filamentary shape with the action of stellar winds of the $\mathrm{O}-\mathrm{B}$ stars driving the $\mathrm{H}$ II regions. A peak of dust temperature at $T=25.8 \mathrm{~K}$ is found right by the position of the BFS57 H II region. The L1649 cloud (Lynds 1962), indicated as [KKY2004]113, is well visible below the filament, but physically unrelated with it, being instead the only prominent feature of the velocity component I in the $\ell 217$ field. Finally, two dense and relatively compact regions are seen in the south. The first one corresponds to S286 and belongs to the velocity component IV $(d=5.3 \mathrm{kpc}, R=10.0 \mathrm{kpc})$, representing the farthest unconfused region detected in this portion of the Hi-GAL survey; in the dust temperature map, it appears warmer $(15 \mathrm{~K} \lesssim T \lesssim 19 \mathrm{~K})$ than the portion of sky it is projected on. The second one (also IRAS06522-0350) is quoted as a satellite star-forming cloud (Megeath et al. 2009) of the G216-2.5 cloud (Lee et al. 1994).

As a global remark, we notice that, invoking the theoretical result of Krumholz \& McKee (2008) about a column density threshold for having MSF, namely $\Sigma=1 \mathrm{~g} \mathrm{~cm}^{-2}$, which, assuming a mean molecular mass of 2.8 , corresponds to $N\left(\mathrm{H}_{2}\right)=2.1 \times 10^{23} \mathrm{~cm}^{-2}$; there are no pixels exceeding this value in our column density map. However, this result cannot be considered resolutive against the presence of MSF in $\ell 217-224$, for at least three reasons: (1) mainly for distant components, possible column density peaks might be diluted in the solid angle subtended by a pixel because of the original instrumental angular resolution and/or the subsequent regridding of the maps for calculating the column density, (2) these column densities and temperatures are derived using the total emission measured along the whole line of sight; however, as it is described below in the paper, if the compact source emission is separated from the background, lower temperatures and higher masses are found for those cores, making it possible to find higher values of the column density, and (3) as shown by Kauffmann \& Pillai (2010), and also suggested by Hi-GAL observations (Elia et al. 

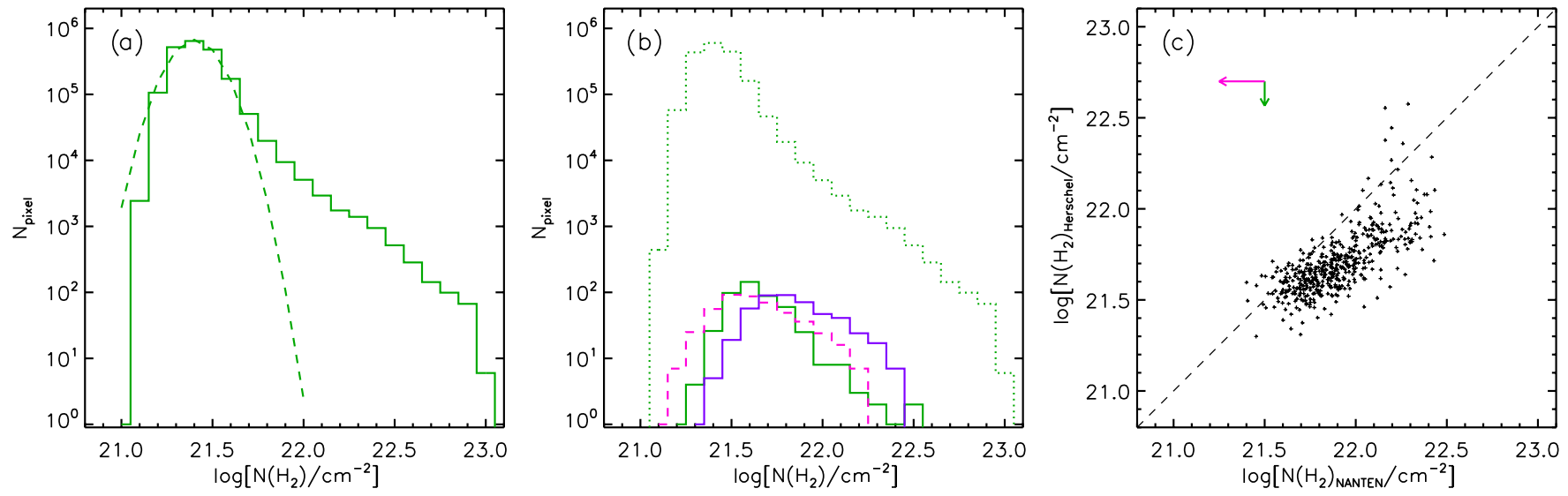

Figure 7. Comparison between the Herschel- and CO-based column densities. (a) Pixel distribution of the original Herschel-based map. The green dashed curve represents the log-normal fit to the Herschel histogram. (b) The green dotted histogram is calculated as in panel (a), but considering only the pixels belonging to the area also covered by the $\mathrm{CO}(1-0)$ map. The green solid histogram is the pixel distribution of the Herschel-based column density map once reprojected on the CO map grid. The purple solid histogram is the distribution of the pixels of CO-based column density map belonging to the common coverage area. The magenta dashed histogram is the same distribution as the previous one, but calculated using the $X$ coefficient of Dame et al. (2001). (c) Pixel-to-pixel plot of the values comprising the dotted histograms of panel (a). The dashed line represents the 1:1 relation. The green and magenta arrows represent the shift to be applied to the plotted points if different $k_{0}$ and $X$ coefficients are employed to compute the column density from the Herschel and the CO maps, respectively (see the text). The $x$-axes of all panels and the $y$-axis of panel (c) are the same for comparison.

(A color version of this figure is available in the online journal.)

2010), the Krumholz \& McKee (2008) critical value probably constitutes too severe a threshold for MSF; this point will be further discussed in Section 5.5.

\subsection{Comparison of the Two Techniques}

The availability of both the $\mathrm{CO}(1-0)$ and Herschel column density estimates allows us to compare the performance of an optically thick spectral line and of the dust as density tracers of the clouds, and to check the validity of the empirical relation (2) in this portion of the Galactic plane. We consider the Herschel-based map (Figure 5) the most reliable of the two, the cold dust being a truly optically thin tracer of the ISM density. The empirical CO-based method may fail in single cases, mainly in the densest parts of the clouds, as recently further demonstrated by Shetty et al. (2011) by means of radiative transfer models. Also, it is sensitive to the excitation conditions of the gas. A systematic characterization of these factors affecting the $\mathrm{CO}$-derived column densities will be reported in a forthcoming paper by Carlhoff et al. (2013).

In Figure 7(a), the histogram of this map is reported. It is characterized by a peak well described by a log-normal behavior (the log-normal fit is also shown), followed by a powerlaw-like extension at higher column densities. This situation is commonly found in regions forming low-mass (Kainulainen et al. 2011) and high-mass (Hill et al. 2011; Schneider et al. 2012) stars, the log-normal shape being attributed to turbulence, and the deviation at large densities due to strongly selfgravitating systems, a typical signature of star formation activity (e.g., Klessen 2000; Federrath et al. 2008). A more quantitative analysis of the power-law tail of this requires separating the different components along the line of sight. However, this is beyond the scope of this paper and will be presented in another study focusing on the probability density functions.

The CO-based column density has been derived for the four distance components through Equation (2), adopting the corresponding $X$ factor values reported in Table 1; subsequently, these four maps have been co-added to obtain the total column density map. To better compare the two maps, we consid- ered only the portion corresponding to the common coverage area (Figure 7(b), green dotted line), and then reprojected the Herschel-based map onto the grid of the $\mathrm{CO}(1-0)$ map, considering only the pixels with meaningful values in both maps (zeros can be found, especially in the CO-based map). The histograms obtained in this way (Figure 7(b), solid lines) become directly comparable, being derived from the same amounts of pixels.

It is possible to appreciate that the histogram of the CO-based column density appears quite different from both the original Herschel-based map and its regridded version. In the first case, the largest CO-based density values are more than half an order of magnitude lower than those revealed in the Herschel-based map. This could be due to the combination of different concomitant effects. First, the $\mathrm{CO}(1-0)$ is not an ideal tracer of the high-density gas distribution. This mainly affects the histogram shape, such as the lack of a tail, for instance, which we observe in the Herschel-based map (e.g., Shetty et al. 2011). Second, the significant pixel size difference between the SPIRE and NANTEN beams (11'.5 against $\left.4^{\prime}\right)$ produces in the latter case a much higher degree of dilution of the local bright peaks in the final pixels, removing from the histogram the high-density tail that is seen instead in the former. This effect can be assessed by considering the histogram of the reprojected Herschel-based map; in this case, the two histograms have more similar peak positions and ranges of variability. However, the regridding of the Herschel-based column density map produces a strong attenuation of both the low- and high-density tails of its histogram, whereas the CO-based map appears to be more populated. This can also be seen in the plot of the Herschel-based column density (reprojected) versus the CO-based one, built pixel-to-pixel and shown in Figure $7(\mathrm{c})$. In the majority of cases $(\sim 92 \%)$ the former is smaller than the latter, and the ratio of the sums of the involved pixels is $\sim 0.6$. This is just a global indication that cannot be taken simply as a sort of further correction of the adopted $X$ factors since the relation between the two distributions does not appear to be linear. The $X$ factor is also known to depend strongly on local variations of the physical conditions because it is expected to be lower in zones of star formation activity (Pineda et al. 2008). Furthermore, the CO line 
intensity depends on the temperature of the gas. However, we can at least evaluate how the choice of the $X$ factor might affect the conclusions achievable by comparing the two column density maps. Adopting $X=1.8 \times 10^{20} \mathrm{~cm}^{-2} \mathrm{~K}^{-1} \mathrm{~km}^{-1} \mathrm{~s}$ (Dame et al. 2001; see Section 3.1), the original CO map histogram is shifted toward lower densities, and its shape gets slightly modified because of different coefficients initially adopted for the components I-IV (magenta dashed histogram in panel (b)). In this case, the distribution peaks at a value smaller than that of the Herschel-based one. On the other hand, a different choice of the $k_{0}$ constant in Equation (4) would produce a rigid shift of the Herschel-based column density histogram. For example, the constant of Preibisch et al. $\left(1993, k_{0}=0.005 \mathrm{~cm}^{2} \mathrm{~g}^{-1}\right.$ at $230 \mathrm{GHz}$ ) can be translated, for $\beta=2$ as in our case, to $0.14 \mathrm{~cm}^{2} \mathrm{~g}^{-1}$ at $1200 \mathrm{GHz}$, producing $40 \%$ lower column densities. These possible corrections are represented in panel (c) as arrows, showing that the choice of a smaller $X$ coefficient would generally produce a better agreement between the two distributions, while a larger dust opacity would obtain the opposite effect.

\section{CORES AND CLUMPS}

In this section, we start to focus on the distribution and the properties of the compact sources detected in the Hi-GAL observations of $\ell 217-224$. The distance estimate for these sources is performed exploiting the kinematic distances of CO clumps.

\subsection{Photometry of Herschel Compact Sources}

The compact source detection and photometry have been performed at each of the five available bands following the procedure described in previous Hi-GAL articles (Molinari et al. 2010a; Elia et al. 2010). We used the Curvature Threshold Extractor package (CuTEx; Molinari et al. 2011), which detects the sources as local maxima in the second-derivative images and then fits an elliptical Gaussian to the source brightness profile to estimate the integrated flux of the source. The parameters of the best fit (the peak position and strength, the minimum and maximum FWHM, and the integral of the Gaussian) are used to estimate the source position, the peak flux, the angular size, and the total flux. The flux uncertainties have been derived from the best fit as well. Using this approach, five independent lists of photometry have been obtained at the five Hi-GAL wavebands.

The completeness limits have been estimated evaluating the CuTEx ability of recovering synthetic compact sources randomly dispersed on the original maps. Both the size and the flux of the generated sources mirror the distributions contained in the original CuTEx lists. The completeness limit at each waveband is then estimated as the flux value at which the histogram of the fraction of the recovered sources exceeds the threshold of $90 \%$ of the total number of injected synthetic sources having fluxes in that bin. The procedure is illustrated in Figure 8 , and the obtained values are $1.75,1.06,0.60,0.53$, and $0.56 \mathrm{Jy}$ at $70,160,250,350$, and $500 \mu \mathrm{m}$, respectively.

Subsequently, the sources extracted in each waveband have been associated by means of a simple spatial criterion (Elia et al. 2010) in order to obtain a band-merged catalog. The total number of entries in this catalog is 3476 , composed by sources having a detection in at least one band. In Section 5, the further selection of a sub-sample of reliable SEDs eligible for the fit through a model is reported.

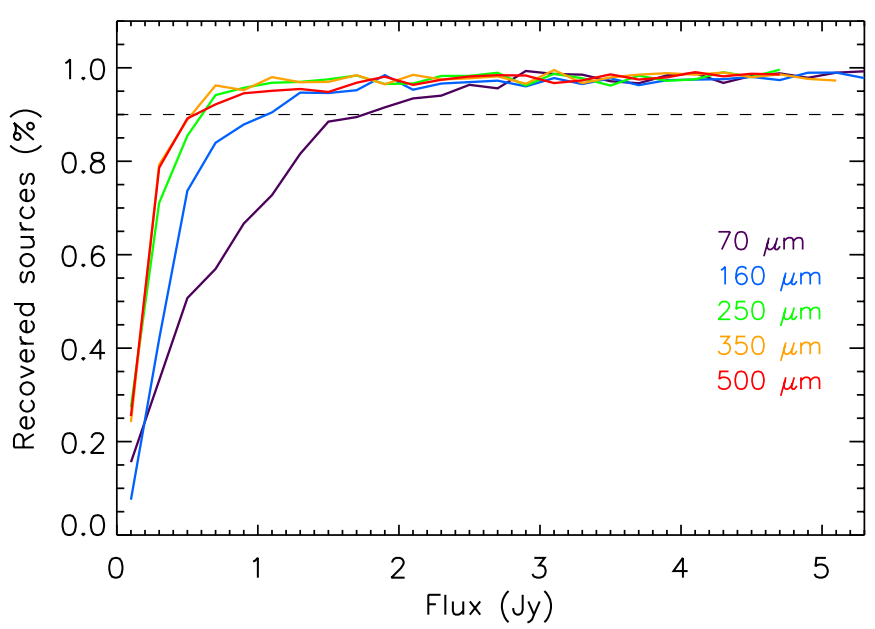

Figure 8. Completeness limit calculation for Hi-GAL photometry. The curves represent the percentage of recovered artificial sources as a function of their fluxes in the five Herschel bands. The dashed horizontal line represents the $90 \%$ limit.

(A color version of this figure is available in the online journal.)

\subsection{Cloud Decomposition}

Similar to the source extraction performed on the Herschel maps, a search for spatially and kinematically coherent overdense structures has been carried out in the $\mathrm{CO}(1-0) l-b-v$ cube, using cloud decomposition techniques. By doing this, we do not aim to find exact counterparts of the Herschel sources or to establish some biunivocal correspondence between the two populations. Rather, due to the coarser spatial resolution of the $\mathrm{CO}(1-0)$ observations and to the different source extraction techniques adopted, the $\mathrm{CO}(1-0)$ coherent sources (hereafter "CO clumps," although in some cases they could not match exactly the definition of clump having typical cloud sizes) are expected to be generally more extended than the Herschel ones, and thus to be found in association with more than one of them in general.

For the cloud decomposition we used the CLOUDPROPS package $^{13}$ (Rosolowsky \& Leroy 2006, 2011), which is also able to derive a set of the basic morphological and physical properties of the detected clumps. We tested both the native clump-finding algorithm present in the package and the widely used cloud decomposition algorithm CLUMPFIND (Williams et al. 1994) which is also implemented in the package as an alternative option. Although CLUMPFIND is known to be strongly dependent on the initial parameter setup, as discussed in detail, e.g., by Brunt et al. (2003), Rosolowsky \& Blitz (2005), Rosolowsky \& Leroy (2006), and Elia et al. (2007), in this case it turned out to be more able than the native CLOUDPROPS search engine to detect structures with the same efficiency across the whole map, probably due to different rms noise conditions throughout the data cube. ${ }^{14}$

The list of the detected clumps is reported in Table 2 in Appendix B, complemented with the following quantities provided by CLOUDPROPS and calculated as described in

\footnotetext{
13 The CLOUDPROPS package is available at https://people.ok.ubc.ca/erosolo/cprops/index.html.

14 In more detail, the native engine of CLOUDPROPS has not been able to detect evident structures in some portions of the map even at very low detection thresholds, which included a number of false detections in other locations. Instead, CLUMPFIND managed to recover all the main features of the data cube using a global and reasonable value of the global threshold $(3 \sigma$; see Williams et al. 1994).
} 
Rosolowsky \& Leroy (2006), with some further tuning of the default settings.

1. Velocity dispersion. It is derived as the second moment of the intensity distribution along the velocity dimension.

2. Distance. To derive the heliocentric distances, the Galactic rotation curve of Brunthaler et al. $\left(2011, R_{0}=8.3 \mathrm{kpc}\right.$, $\Theta_{0}=239 \mathrm{~km} \mathrm{~s}^{-1}$ ) has been used instead of the default one of Reid et al. (2009) implemented in CLOUDPROPS.

3. Radius. The deconvolved radius $r$ is estimated as the geometric mean of the deconvolved major and minor axes of the clump, given in units of pc once the clump distance is known.

4. Mass. The mass of the clump $M$ is obtained as in Section 3.1, i.e., integrating the column density over the solid angle of the clump. The column density is derived by CLOUDPROPS as in Equation (2) with an $X$ factor constant for all clumps. We then rescaled the mass considering an $X$ factor varying with the Galactocentric distance as in Equation (3).

5. Virial mass. The virial mass is calculated as $M_{\mathrm{vir}}\left[M_{\odot}\right]=$ $1040 \sigma_{v}^{2}\left[\mathrm{~km} \mathrm{~s}^{-1}\right] r[\mathrm{pc}]$, under the assumption that each clump is spherical and virialized with a density profile of the form $\rho \propto r^{-1}$ (Solomon et al. 1987). Density power laws with exponents 0 and -2 would require, instead, a constant of 1150 and $690 M_{\odot}\left(\mathrm{km} \mathrm{s}^{-1}\right)^{-1} \mathrm{pc}^{-1}$, respectively.

6. Luminosity. The clump luminosity in the $\mathrm{CO}(1-0)$ line is provided in units of $\mathrm{K} \mathrm{km} \mathrm{s}^{-1} \mathrm{pc}^{2}$. The conversion factor to solar luminosities for this transition is $4.9 \times$ $10^{-5} L_{\odot}\left[\mathrm{K} \mathrm{km} \mathrm{s}^{-1} \mathrm{pc}^{2}\right]^{-1}$ (Solomon et al. 1992).

After ruling out sources having meaningless physical parameters (corresponding to failures of the CLOUDPROPS source extraction) the final list is composed of 321 clumps.

\section{HERSCHEL SOURCES: PHYSICAL PROPERTIES}

\subsection{SED Building}

In this section, we derive the basic physical properties of the Hi-GAL compact sources from their SEDs. However, first of all, a selection has to be performed in order to obtain a reliable sample of SEDs eligible for the modified blackbody fit.

Where present, the flux at $70 \mu \mathrm{m}$ is not considered in this fit, being generally affected by the direct contribution of a proto-stellar component; for this reason, its presence can be used as a signature to distinguish a proto-stellar from a starless core/clump (e.g., Bontemps et al. 2010; Giannini et al. 2012). We also searched for possible WISE $22 \mu \mathrm{m}$ counterparts (detected at $\mathrm{S} / \mathrm{N}>5 \sigma$ ) within a searching radius of $12^{\prime \prime} .6$ (corresponding to the WISE resolution at that wavelength and to the WISE and Herschel positional uncertainties combined in quadrature) around the Hi-GAL source coordinates. The low $\mathrm{S} / \mathrm{N}$ in our PACS $70 \mu \mathrm{m}$ maps, responsible for the large flux completeness limit at this band, can result in an association between a WISE source and a Herschel one lacking emission at $70 \mu \mathrm{m}$. In these cases, the association is considered genuine and the source is classified as proto-stellar if at least the flux at $160 \mu \mathrm{m}$ is available. In this way, 236 out of $943 \mathrm{Hi}$-GAL sources with a selected SED (see below) are found to be associated with a WISE counterpart.

To estimate which fraction of the WISE-Herschel associations could be spurious, we generated a random distribution of coordinate pairs having the same number and spatial coverage of the WISE sources in our field, and ran the same procedure of matching with the Hi-GAL sources. Only 18 of the aforementioned $943 \mathrm{Hi}-\mathrm{GAL}$ sources are associated with a source generated in such a way, i.e., less than $8 \%$ if compared with the number of the WISE counterparts found. This percentage represents the occurrence of spurious matches that should be expected to affect our WISE-Herschel association.

At the longer wavelengths, we deal with the frequent case of the increase of the beam-deconvolved diameter, as estimated by $\operatorname{CuTEx}\left(\theta_{\lambda}=\sqrt{\mathrm{FWHM}_{\text {maj, } \lambda} \times \mathrm{FWHM}_{\min , \lambda}-\mathrm{HPBM}_{\lambda}^{2}}\right)$, at increasing wavelengths, already discussed by Motte et al. (2010) and Giannini et al. (2012). Due to this increase of the area over which the emission is integrated, many SEDs flatten or even rise longward at $\lambda \geqslant 250 \mu \mathrm{m}$.

Here we adopt the strategy of Motte et al. (2010), consisting of assuming a reference wavelength to estimate the source angular size, and scaling the fluxes at a larger wavelength $\lambda$ by the ratio between the deconvolved sizes at the reference wavelength and at $\lambda$, respectively. Due to the further selection to identify SEDs eligible for the fit, described at the end of this section, the shortest wavelength always available is $250 \mu \mathrm{m}$ which we adopt as the reference wavelength:

$$
\bar{F}_{\lambda}=F_{\lambda} \times \frac{\theta_{250}}{\theta_{\lambda}}, \quad \lambda \geqslant 350 \mu \mathrm{m} .
$$

This is based on the assumptions that (1) the source is optically thin at $\lambda \geqslant 250 \mu \mathrm{m}$, (2) the temperature gradient is weak (Motte \& André 2001), and (3) the radial density profile of the source is described by $\rho(r) \propto r^{2}$, then $M(r) \propto r$.

It is noteworthy that scaling fluxes in this way corresponds to considering fluxes emitted by the same volume of dust which is more compatible with fitting a modified blackbody to an SED. On the other hand, hereafter it must be kept in mind that the physical properties derived from the fit of the scaled SEDs describe the average conditions of the source inside a volume delimited by the size detected at the reference wavelength. Finally, considering a smaller volume, we reduce the influence of possible multiplicities (i.e., shorter wavelength counterparts that appear confused in an unresolved source at 350-500 $\mu \mathrm{m}$ ) on the fluxes composing the final SED.

A further selection has been performed on the SEDs built as described above, accepting only those (1) being composed by consecutive fluxes (at least three), (2) showing no dips (e.g., concave shape), (3) not peaking at $500 \mu \mathrm{m}$, and (4) having a distance estimate, as described in Section 5.2.

After applying these constraints, we finally obtained a sample of 943 SEDs eligible for the fit, 255 of which are proto-stellar (184 of which detected at both $22 \mu \mathrm{m}$ and $70 \mu \mathrm{m}, 52$ only at $22 \mu \mathrm{m}$, and 19 only at $70 \mu \mathrm{m}$ ) and 688 of which are starless.

The positions of these sources in the surveyed region are reported in Figure 9, where we introduce the color convention blue for proto-stellar objects and red for starless ones. We remark that some bright sources might have been ruled out from the present statistical analysis by the constraints we applied to deal with regular SEDs.

Furthermore, we draw attention to the fact that many sources appear to lie on the most prominent filaments present in the maps. Although these filaments appear uniform in figures like those shown in Appendix A due to the choice of the intensity scale, they actually possess a complex internal structure revealed by Herschel and characterized by the presence of numerous compact sources (see, e.g., Molinari et al. 2010a). This aspect is well exemplified by Figure 10, where the positions of the sources extracted at $250 \mu \mathrm{m}$ in the neighborhoods of the 


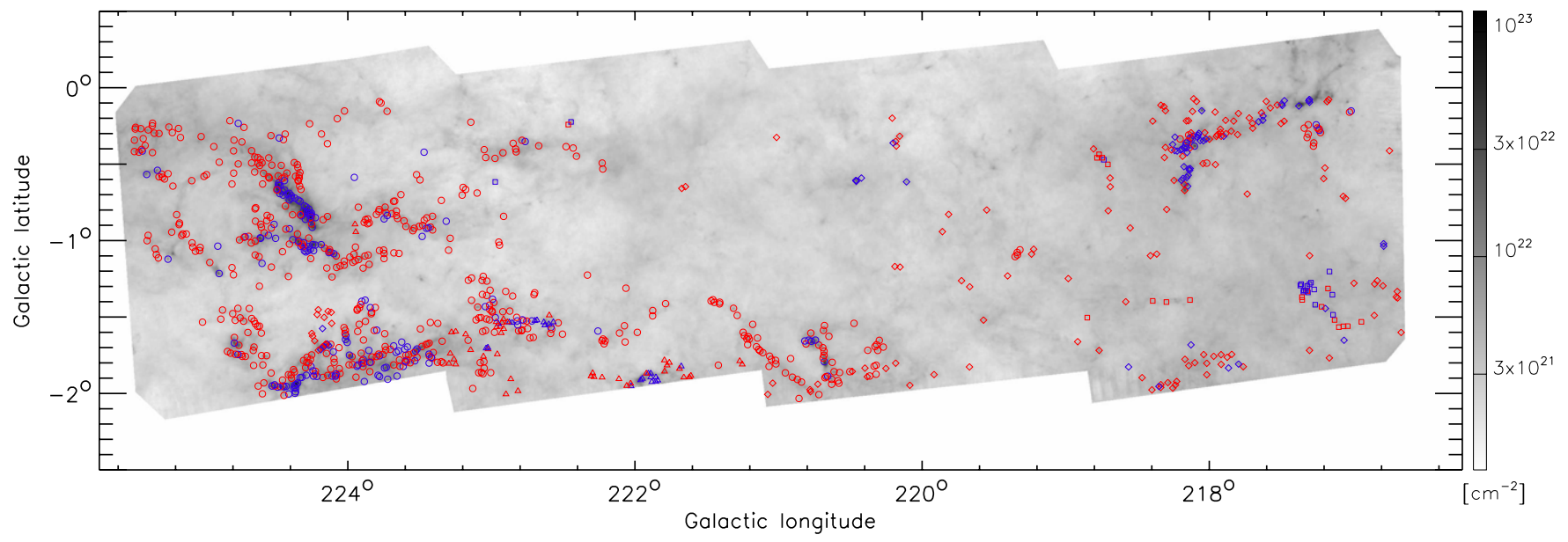

Figure 9. Spatial distribution of the Hi-GAL compact sources considered for the analysis in this paper (see the text), overplotted on the column density map of Figure 5. Different symbols are used for sources associated with the four CO components identified in Section 3.1: circles, triangles, diamonds, and squares correspond to the components I, II, III, and IV, respectively. The blue and red colors are used to indicate proto-stellar and starless sources, respectively.

(A color version of this figure is available in the online journal.)
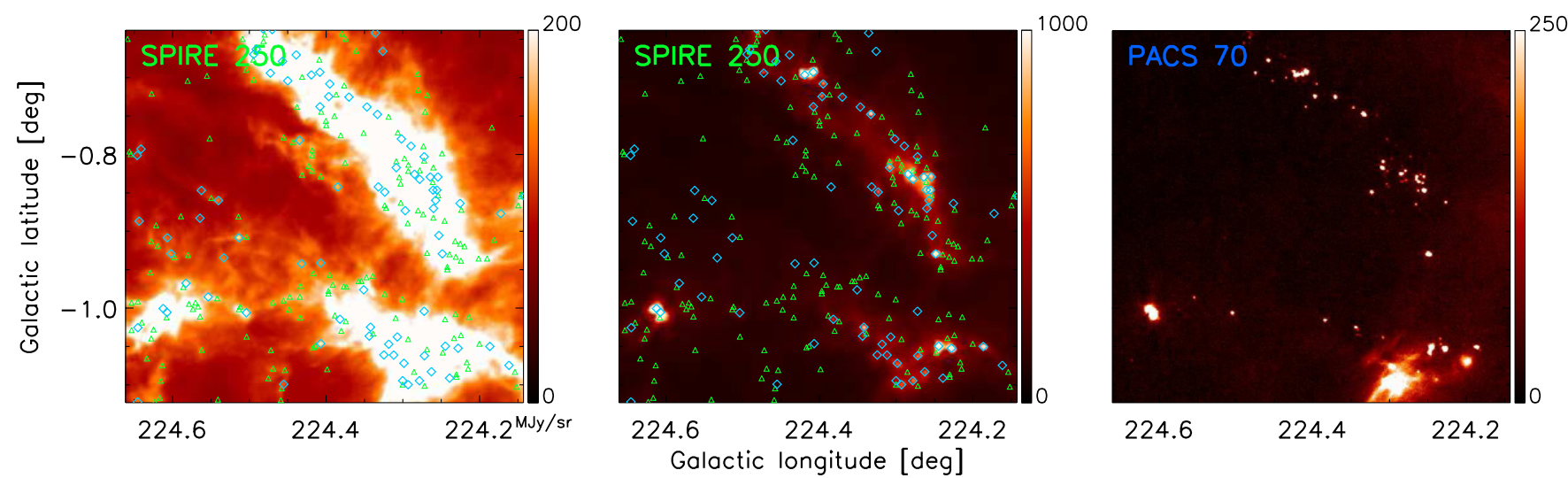

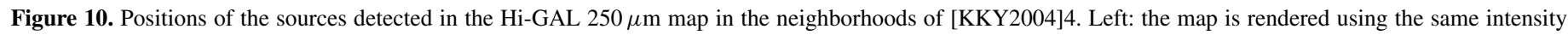

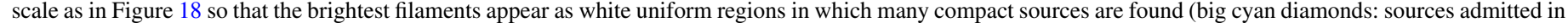

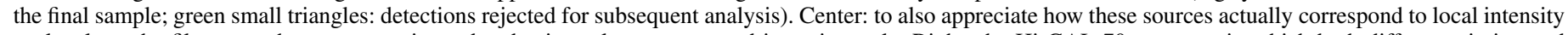

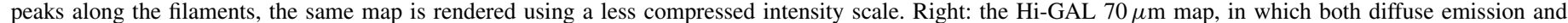

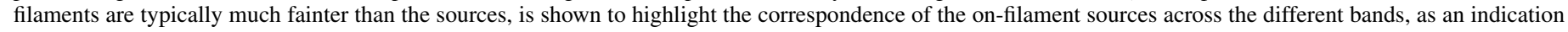
of their genuineness.

(A color version of this figure is available in the online journal.)

[KKY2004]4 cloud are superimposed on the map of this wavelength rendered by means of two different intensity scales. The first one is appropriate to show the appearance of the diffuse emission, while the second one is needed to reveal the internal structure of the bright filaments where it is possible to find compact sources whose genuineness is confirmed, for example, by comparison with the $70 \mu \mathrm{m}$ map. The occurrence of false positives in our extraction cannot be excluded a priori. However, since the detection is performed independently at each waveband, it is unlikely to systematically find counterparts at other wavelengths. In any case the source selection described in this section, based on the regularity of the collected SED, ensures the reliability of the sources considered for the subsequent analysis.

\subsection{Distance Assignment}

The problem of the distance estimation of the Hi-GAL sources is a crucial point that has to be faced to derive a complete and meaningful set of physical properties for these objects. Fortunately in the portion of the Galactic plane hosting the $\ell 217-224$ field this issue turns out to be less problematic, thanks to both the low degree of confusion along the line of sight and the absence of the near/far ambiguity on the kinematic distance, as pointed out in Section 3.1.

A possible approach might consist of examining the line of sight in the $\mathrm{CO}(1-0)$ data cube corresponding to an Hi-GAL source, and to take the $v_{\text {lsr }}$ of the brightest component encountered, as done, for example, in Russeil et al. (2011). Here, however, we want to exploit the additional information offered by the $\mathrm{CO}(1-0)$ data cube decomposition in clumps (Section 4.2): in the $\mathrm{CO}(1-0)$ line of sight containing the source, we consider the spectral channels assigned by CLOUDPROPS/CLUMPFIND to one or more clumps, and associate with the source the distance of the clump, if any, having the closest centroid. There is a double advantage to adopting this strategy: first, the distance assigned to all the sources associated with a given clump is the same, without finding a different distance at each spatial/spectral position in the $\mathrm{CO}(1-0)$ cube, perhaps affected by the clump internal kinematics unrelated to Galactic rotation. Second, this strategy acts quite efficiently in cases of the presence, along the same line of sight, of a peripheral $\mathrm{CO}(1-0)$ feature of a large and bright clump and of a 

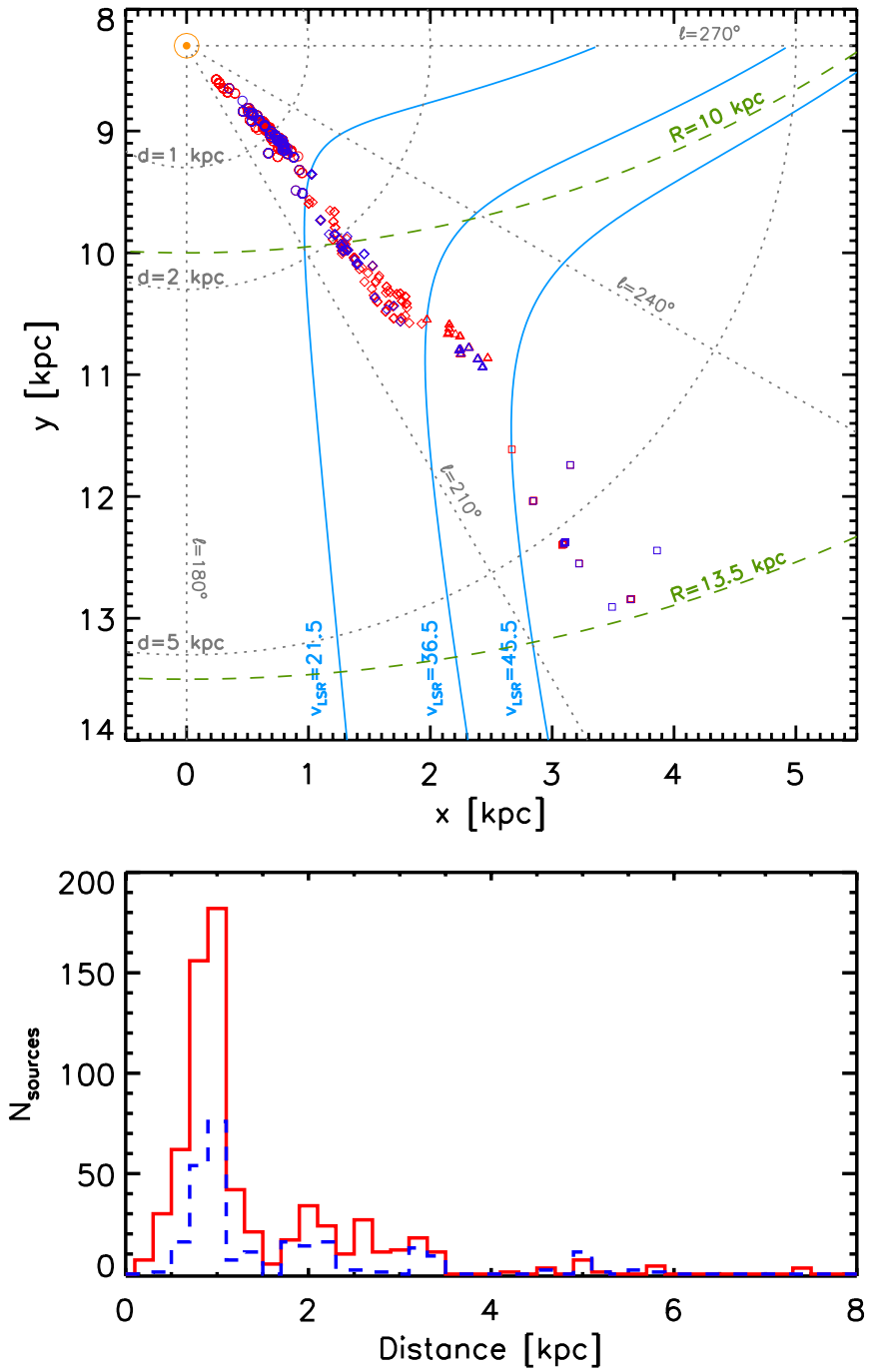

Figure 11. Top: sketch of the TGQ containing the positions of the $\ell 217-224$ sources selected for the SED fit. The origin of the axes is in the Galactic center, and $x$ and $y$ designate the components of the Galactocentric distance (with $x$ and $y$ increasing toward the direction of $\ell=270^{\circ}$ and $\ell=180^{\circ}$, respectively). The symbol and color conventions are the same as that in Figure 9. The orange $\odot$ symbol represents the Galactic position of the Sun. The gray dotted lines indicate relevant longitude values, reported in the labels of the same color. The gray dotted and green dashed arcs represent relevant values of heliocentric and Galactocentric distance, respectively. Finally, the cyan solid lines are isotachs corresponding to the three $v_{\mathrm{lsr}}$ values separating the four main $\mathrm{CO}(1-0)$ components we identified in this paper for convenience. Bottom: histograms of the source heliocentric distances of the sources shown in the upper panel (blue for proto-stellar and red for starless, respectively). The bin width is of $200 \mathrm{pc}$.

(A color version of this figure is available in the online journal.)

weaker but central feature of a smaller clump; in this case, we believe that the Herschel source is more likely associated with the small clump being centered on it, rather than with the outer part of a large clump. Taking into account only the brightest line in this example, instead, would have led to a completely different association.

The distance information can be exploited to generate a view of the spatial distribution of the sources in the Galactic plane (Figure 11, top panel). In this figure we notice a certain degree of continuity in the distribution of the heliocentric distances, without significant gaps between the components I and II. To better illustrate how this classification works, the isotachs corresponding to the velocities conventionally separating the four components are also plotted. According to the spiral arm loci from Cordes \& Lazio (2002), ${ }^{15}$ in the longitude range we consider in this paper the Local arm should be encountered at $d \sim 0.8 \mathrm{kpc}$, the Perseus arm at $d \sim 3 \mathrm{kpc}$, and the outer arm at $d>9 \mathrm{kpc}$.

Our results, in the limit of the reliability of the adopted rotation curve in this part of the Galaxy, partially confirm this view. Looking at the bottom panel of Figure 11, the highest concentration of component I sources is found in the bins centered at 0.8 and $1 \mathrm{kpc}$. Instead, the distance distribution of the component II sources peaks around 2, 2.6, and $3.2 \mathrm{kpc}$, suggesting instead the presence of an inter-arm bridge of matter similar to those found by Carraro et al. (2005) in other locations of the TGQ. The 0.7-1.1, 2.0, 2.6, and $3.2 \mathrm{kpc}$ distances also correspond with the global distances (photometric or kinematic) assigned by Kim et al. (2004) to their clouds present in our field. The outer arm is not detected in this portion of the TGQ, in agreement with Carraro et al. (2005) and $\mathrm{Xu}$ et al. (2009). Finally, we notice that none of the sources we found at the farthest Galactocentric distances can be classified as belonging to the far outer Galaxy ( $d>15 \mathrm{kpc}$; Heyer et al. 1998).

\subsection{SED Fitting}

As stated above, a modified blackbody (described by Equation (4), but with the terms $N\left(\mathrm{H}_{2}\right) \Delta \vartheta_{500}^{2}$ replaced by $\left.M / d^{2}\right)$ has been fitted to the SEDs, to obtain concomitant estimates of the mass and the temperature for each individual source.

In Table 3 the astrometric, photometric, and physical properties of the analyzed sources are resumed. Here we start discussing the physical diameter and the temperature (Figure 12). For sources provided with a distance estimate, the diameter $D$ is easily obtained from the source deconvolved FWHM estimated at $250 \mu \mathrm{m}$. The histogram in panel (a) shows that our sample is composed of a mixture of cores $(D<0.1 \mathrm{pc})$ and clumps ( $D>0.1 \mathrm{pc})$, according to the typical definitions of these object classes based on their size (e.g., Bergin \& Tafalla 2007). It is important, therefore, to keep in mind that a relevant fraction of the compact sources analyzed in this work do not correspond to progenitors of single star systems but, rather, to distant complex and large structures we cannot resolve (in particular, 153 out of 255 proto-stellar sources must be classified as clumps).

The two size distributions do not appear much different from each other $(\langle D\rangle=0.16 \mathrm{pc}$ in both cases), unlike in Giannini et al. (2012) where proto-stellar sources were found to be on average smaller than the starless ones. To better discuss this point, we notice that sources spread on a wide range of distances are used altogether to build the histograms for both the samples, so that some large proto-stellar clumps located at long distances are obviously larger than closer pre-stellar cores. For this reason, we also show the distributions of the diameters as they would appear if placed at a distance of $1000 \mathrm{pc}$ (panel (b)). Also in this case, there is no clear indication of significant size differences. In fact, Giannini et al. (2012) adopted a more flexible criterion to decide the reference wavelength at which the source size is estimated: $160 \mu \mathrm{m}$ when a detection at this wavelength were available, and $250 \mu \mathrm{m}$ otherwise. Since many starless sources are not detected at $160 \mu \mathrm{m}$, their size was estimated at $250 \mu \mathrm{m}$, where it appears generally larger (Section 5.1), thus favoring

\footnotetext{
15 In this case we cannot invoke the careful and more recent spiral arm determinations by Vallée (2005) because they were obtained based on a Galactic rotation curve very different $\left(R_{0}=7.9 \mathrm{kpc}\right)$ from the one we adopt here.
} 

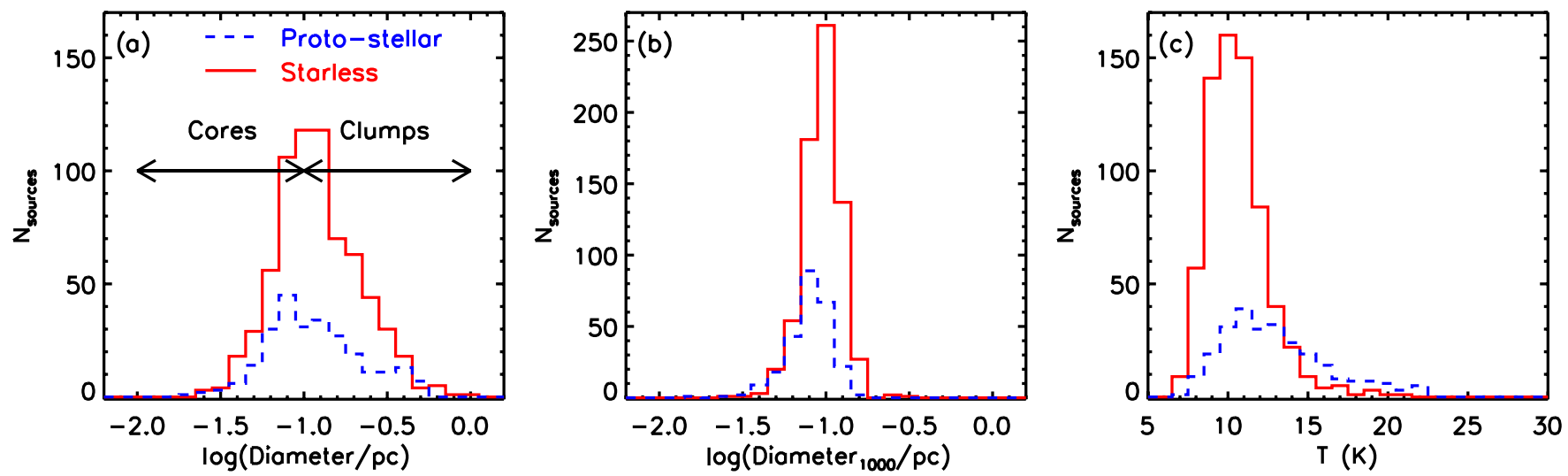

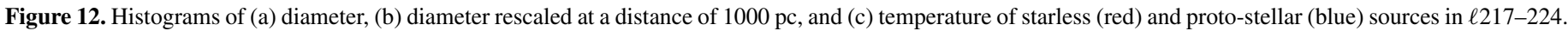
(A color version of this figure is available in the online journal.)

the observed segregation in size, while in our case the reference wavelength is the same in all cases.

Concerning the temperature distributions of the two classes, also in this case the dichotomy proto-stellar-warmer against starless-colder is not evident as in Giannini et al. (2012), again due to the different reference size adopted in that case implying a more consistent flux scaling at the SPIRE wavelengths for the proto-stellar sources, finally leading to higher temperature and lower mass estimates. In our case, the uniform choice of the size at $250 \mu \mathrm{m}$ as reference reduces the differences of the two distributions that can be found in the presence of a significant high-temperature tail for the proto-stellar sources, and in the average temperatures $\left(\left\langle T_{\mathrm{ps}}\right\rangle=13.6 \mathrm{~K},\left\langle T_{\mathrm{sl}}\right\rangle=11.1 \mathrm{~K}\right)$. It is worth noting that the temperature is estimated on the range $160-500 \mu \mathrm{m}$ : this implies that the difference between protostellar and starless sources is independent of the presence of a flux at $70 \mu \mathrm{m}$, i.e., the constraint we impose to classify the protostellar sources. Finally, note that, unlike the size distribution, in this case there is no distance effect biasing the observed trend of the temperature.

\subsection{An Estimate of the Clump and Star Formation Efficiency}

The combination of Hi-GAL and NANTEN data allow us to provide an estimate of the clump and star formation efficiency (CFE and SFE, respectively) for the four distance components seen toward $\ell 217-224$. Indicating the total mass of the compact sources (both proto-stellar and starless) with $M_{\mathrm{cl}}$ and the total cloud mass with $M_{\mathrm{gas}}$, the CFE is expressed by $\mathrm{CFE}=\left(M_{\mathrm{cl}} / M_{\mathrm{cl}}+M_{\mathrm{gas}}\right)$. The cloud masses can be easily derived from the column density map of Figure 5, resolving the infrequent cases of overlap of two distance components along a CO line of sight by adopting the distance corresponding to the brightest feature in the spectrum. Due to the different spatial coverage of the Hi-GAL and NANTEN observations, for each component we again derive the cloud mass only in the lines of sight falling inside the common area. For the same reason, to compute $M_{\mathrm{cl}}$ we must consider only the Hi-GAL sources lying in the area surveyed in the $\mathrm{CO}(1-0)$ line, but all the sources considered for the present analysis fulfill this requirement because they are required to have an associated kinematic distance. Since the column density and the compact sources' masses have been computed using the same opacity $k_{0}$, our CFE will not be affected by the large uncertainties associated with the choice of this constant. The obtained CFEs for the four components are $0.055 \pm 0.008,0.024 \pm 0.003,0.07 \pm 0.01$, and $0.027 \pm 0.004$, respectively. The uncertainties have been estimated assuming a conservative error of $10 \%$ on both the source masses and pixel-to-pixel column density, combined in quadrature.

In the same way, if $M_{*}$ is the total mass of the formed stars, the SFE is expressed by SFE $=\left(M_{*} / M_{*}+M_{\mathrm{gas}}\right)$. To compute $M_{*}$ we consider only the Hi-GAL proto-stellar cores lying in the area surveyed in the $\mathrm{CO}(1-0)$ line, obtaining core total masses of $2880,2540,2140$, and $2550 M_{\odot}$ for the four components, respectively. Finally, these values have been converted into stellar masses assuming a core-to-star conversion efficiency $\varepsilon_{\text {eff }}=1 / 3$ (Alves et al. 2007; André et al. 2010), and average SFEs of $8_{-1}^{+2} \times 10^{-3}, 3.9_{-0.6}^{+0.9} \times 10^{-3}, 1.2_{-0.2}^{+0.3} \times 10^{-2}$, and $5.8_{-0.8}^{+1.2} \times 10^{-3}$ have been obtained in the four cases. Here the relative uncertainties are not simply the same associated with the CFE, since one must take into account also the assumptions on the $\varepsilon_{\text {eff }}$. Following Kauffmann \& Pillai (2010), we adopt $\varepsilon_{\text {eff }}=1 / 2$ as upper limit for this coefficient.

Further considerations, however, lead us to take these estimates as lower limits for the real SFE: (1) this derivation of the SFE is based only on FIR/sub-mm observations, neglecting the mass of more evolved YSOs detected only in the medium infrared, (2) as it will be discussed in Section 6, it is possible that a small fraction of sources classified as starless cores, not taken into account for this calculation, may already have a strongly embedded early-stage proto-stellar content.

On the other hand, at increasing distances the so-called compact sources correspond to structures having increasing physical sizes, departing from the definition of core. Therefore, unresolved distant regions are considered as a single source, although in most cases they contain a complex internal substructure consisting of dense cores and diffuse matter. In particular, for such sources classified as proto-stellar clumps, only a fraction of the mass is taking part in the ongoing star formation processes; nevertheless the total mass is entirely taken into account in the SFE calculation.

In any case, these results are comparable to typical SFE values. In the 40 star-forming complexes analyzed by Murray (2011), the SFE ranges from 0.002 to 0.2 , with an average of 0.08. In Lada et al. (2010), who analyzed 11 nearby molecular clouds, it ranges from $\sim 0.003$ (Pipe nebula) to $\sim 0.09$ (RCrA). Compared with these data, the SFE of our four components can be classified as relatively low. It is noteworthy, in this sense, that we find the lowest SFE in what we call component II, which is likely spatially related to Maddalena's cloud, i.e., one of the most striking cases of giant molecular clouds with an 


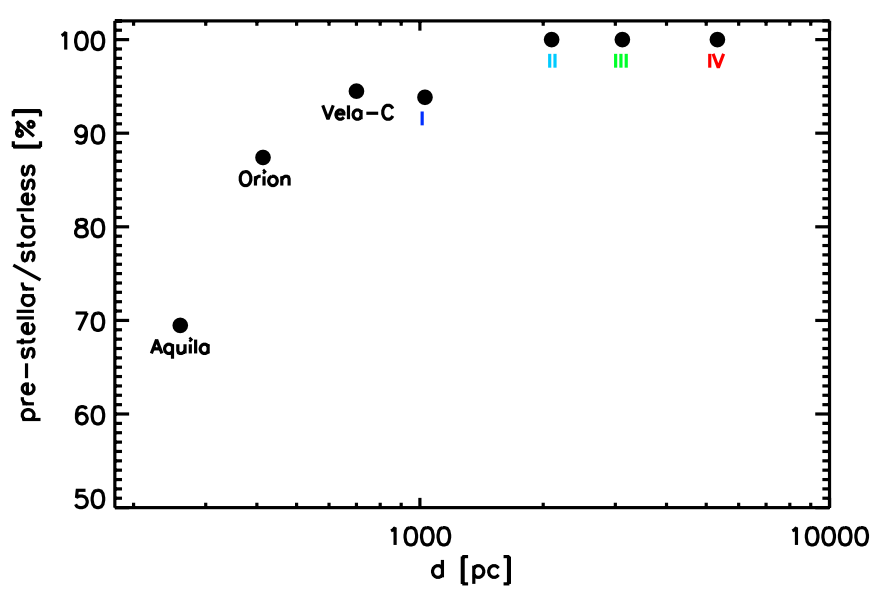

Figure 13. Fraction of pre-stellar sources over the total number of starless ones for seven different regions, as a function of the average region distance. Data from the previous literature have labels in black, while data from this paper have labels with different colors, according to the color-component convention adopted in Figure 4.

(A color version of this figure is available in the online journal.)

unusual combination of high gas mass and little evidence for star formation (e.g., Heyer et al. 2006; Megeath et al. 2009).

\subsection{Mass Distribution of the Sources}

In the following discussion we focus on the starless objects that are also gravitationally bound, which we call pre-stellar cores, because they may give rise to the formation of protostellar clusters. In the recent Herschel literature, to study the stability of such sources, the lack of kinematic information has forced the adoption of the critical Bonnor-Ebert (BE) mass as a surrogate for the virial mass (Könyves et al. 2010; Giannini et al. 2012). We also use it in the present case; although, in principle, we could exploit the velocity dispersion obtainable by the $\mathrm{CO}(1-0)$ data to estimate the virial mass, in practice, the $\mathrm{CO}(1-0)$ line spectral resolution is too coarse for determining reliable line widths.

The BE mass is given by

$$
M_{\mathrm{BE}}=2.4 r_{\mathrm{BE}} a^{2} / G
$$

where $a$ is the sound speed at the source temperature, $G$ is the gravitational constant, and $r_{\mathrm{BE}}$ is the $\mathrm{BE}$ radius (in pc), which in this case is assumed to coincide with the source radius observed at the reference wavelength. We consider gravitationally bound, and therefore pre-stellar, to encompass all the starless sources with $M>M_{\mathrm{BE}}$ (cf. Olmi et al. 2010; Giannini et al. 2012). According to this definition, we find 398, 131, 42, and 19 prestellar sources associated with the gas velocity components I, II, III, and IV, corresponding to $82 \%, 94 \%, 98 \%$, and $100 \%$ of the starless core populations, respectively.

In our case, the availability of four different distance components gives us the chance to discuss a trend emerging from the recent Herschel literature. The studies of the relatively nearby star-forming regions of Aquila (Könyves et al. 2010), Orion-A (D. Polychroni et al. 2013, in preparation), and Vela-C (Giannini et al. 2012), located at 260, 414, and 700 pc away, respectively, provided an estimate of the pre-stellar fraction over the total starless core number of $69 \%, 87 \%$, and $94 \%$, respectively. These percentages have been estimated using a less severe threshold for identifying the gravitationally bound sources, namely $M>0.5 M_{\mathrm{BE}}$. If we adopt the same criterion for the components I-IV of the present work, the fraction of pre-stellar sources

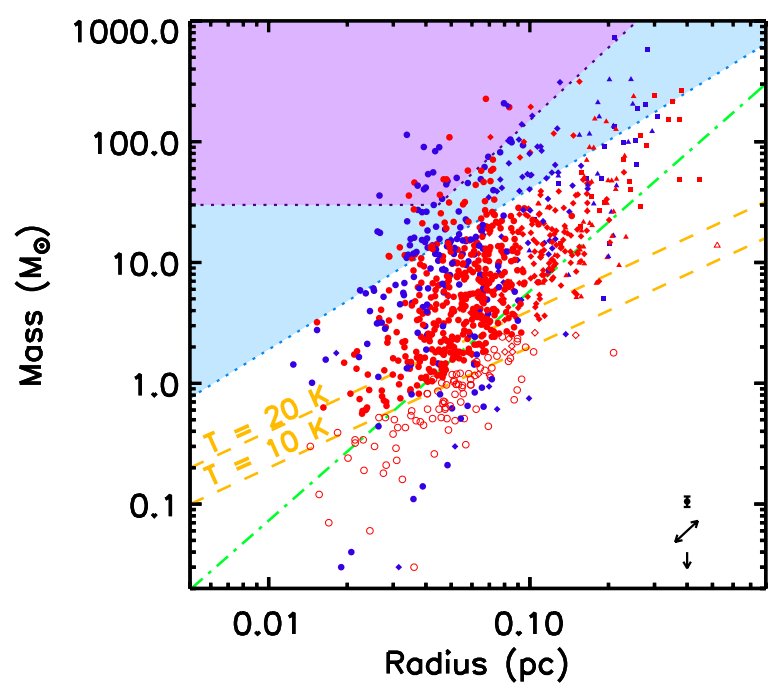

Figure 14. Mass vs. radius diagram for the Hi-GAL sources in $\ell 217-224$. Symbols with the same shape convention of Figure 9 represent pre-stellar (red filled), starless unbound (red open), and proto-stellar (blue filled) sources. Orange dashed lines are the loci of sources with $M=1 M_{\mathrm{BE}}$, for $T=10 \mathrm{~K}$ and $T=20 \mathrm{~K}$, while the green dot-dashed line represents the power law estimated by Larson (1981). The area with a purple background represents the region corresponding to the conditions for MSF to occur, according to the Krumholz $\&$ McKee (2008) threshold (purple dotted line). It represents a sub-region of the blue-background area, corresponding to the MSF threshold of Kauffmann \& Pillai (2010, blue dotted line). In the bottom-right corner of the plot, three possible sources of uncertainty are represented: the error bar represents a $10 \%$ uncertainty on the mass coming from the original photometry, the double arrow indicates the shift that would be produced by a $\pm 10 \%$ uncertainty on the distance, and the down arrow indicates the shift that would be applied if a different opacity law was adopted, as proposed in Section 3.3.

(A color version of this figure is available in the online journal.)

increases to $94 \%, 100 \%, 100 \%$, and $100 \%$, respectively. We notice a general trend of this fraction to increase with the distance (see Figure 13), highlighting a clear selection effect against gravitationally unbound structures located at large distances (at $d \geqslant 2000 \mathrm{pc}$ the saturation is practically achieved). In fact, this is implicit in Equation (6): given a certain SED and changing its distance $d, M$ varies as $d^{2}$, while $r_{\mathrm{BE}}$ varies as $d$, thus making it easier for the same SED to fulfill the BE criterion when $d$ increases. On the one hand, this means that to exploit this fraction of gravitationally bound sources in a region as an evolutionary indication about its star formation capability is affected by a strong and practically unsolvable bias related to the distance. On the other hand, the slight deviation of the behavior of the component I from the general trend seen in Figure 13 can be considered, from the qualitative point of view, indicative of an intrinsically lower occurrence of pre-stellar sources compared, for example, with the Vela-C region.

A mass versus radius plot, shown in Figure 14, can be used to illustrate and quantify the discussion of the gravitational stability of the starless cores (neglecting the turbulence and magnetic field supports against gravity). The lines corresponding to $1 M_{\mathrm{BE}}$ mass behavior at two different temperatures, $T=10 \mathrm{~K}$ and $20 \mathrm{~K}$, respectively, are displayed (orange dashed lines). It can be seen that all the unbound sources (red open symbols) lie below the curve at $T=20 \mathrm{~K}$, while, at the same time, most of the bound ones (red filled symbols) show a remarkable degree of boundedness (i.e., $M \gg M_{\mathrm{BE}}(T=20 \mathrm{~K})$ ). Although this diagram is meaningless in the case of the proto-stellar sources, since part of the mass has already formed the central protostar(s) or could have been ejected in the form of proto-stellar 
jets, we report also these sources in the plot (blue filled symbols) for completeness.

In Figure 14, "Larson's third law" is shown as a green dotdashed line. Originally formulated as $M(r)>460 M_{\odot}(r / \mathrm{pc})^{1.9}$ (Larson 1981) it was later revised: Solomon et al. (1987) found a value of 2 for the power-law exponent, i.e., a constant column density as a function of the radius. Since the two values are very similar, we plot this relation in its original form. In any case, it can be seen that the sources of our sample do not obey a precise power law. Therefore, we do not try here to fit a power law to the mass-size distribution of the pre-stellar cores in $\ell 217-224$.

The mass versus radius diagram is also a useful tool for checking the presence of the conditions for MSF, based on the intuitive concept that to form massive stars a conspicuous mass reservoir concentrated in a relatively small volume is required. In the recent literature, a theoretical column density threshold of $1 \mathrm{~g} \mathrm{~cm}^{-2}$ (Krumholz \& McKee 2008) is largely invoked and used to identify candidate sites of MSF (see also Section 3.2). It can be represented as a locus in the bi-logarithmic plot of Figure 14, down to a minimum core mass value that, for a $10 M_{\odot}$ star and a core-to-star conversion efficiency $\varepsilon_{\text {eff }}=1 / 3$, amounts to $30 M_{\odot}$. A small but significant fraction of sources (both pre- and protostellar) exceed this threshold and can therefore be considered potential progenitors of massive stars. Also, an empirical and less demanding limit for MSF has been estimated by Kauffmann \& Pillai (2010), based on observations of Infrared Dark Clouds (IRDCs; Egan et al. 1998), as $M(r)>870 M_{\odot}(r / p c)^{1.33}$ and reported in Figure 14. Further sources, belonging to all the four distance components of $\ell 217-224$, fulfill this threshold, testifying the occurrence of the conditions for having MSF also in this portion of the TGQ and, in particular, at remarkably large Galactocentric distances ( $R>11 \mathrm{kpc}$ for the component IV).

Moving to the analysis of the mass distribution, it becomes necessary to deal with homogeneous samples, from both the spatial and the evolutionary point of view. Thus, we consider only the pre-stellar sources of the closest velocity component (I), a choice that also ensures enough statistics to obtain a reliable mass distribution. Again, it is correct to calculate this kind of distribution only for the pre-stellar sources, as the masses of the proto-stellar ones are not representative of the initial core mass.

The core mass distribution is reported in Figure 15. The mass completeness limit of $1.9 M_{\odot}$ has been estimated from the flux completeness limit at the reference wavelength of $250 \mu \mathrm{m}$ (Section 4) using the modified blackbody equation, combined with the average temperature and distance of the sample, and $\beta=2$. The turnover of the mass function falls around $3 M_{\odot}$, too close to the completeness limit to claim that we are well tracing the log-normal behavior of the mass distribution below the turnover point (as, e.g., in Könyves et al. 2010). We then fit a power law $N(\log M) \propto M^{\alpha}$ to the high-mass part of the distribution using an approach based on the method of maximum likelihood and independent of the histogram binning (see Olmi et al. 2013 and references therein). It allows us to determine the lower limit of range of validity of the power law, $\log \left(M_{\text {inf }} / M_{\odot}\right)=0.65$, and its slope $\alpha=-1.0 \pm 0.2$.

Whereas the core mass functions that are derived for nearby regions through spectral lines (Orion A, $\alpha=-1.3 \pm 0.1$; Ikeda et al. 2007), Spitzer (Perseus, Serpens, and Ophiuchus, $\alpha=-1.3 \pm 0.2$; Enoch et al. 2008) and Herschel observations (Aquila Rift, $\alpha=-1.45 \pm 0.2$; Könyves et al. 2010) have slope values consistent with those of the stellar initial mass function $(\alpha=-1.3 \pm 0.7$ for single stars, Kroupa 2001; $\alpha=-1.35 \pm 0.3$ for multiple systems, Chabrier 2005), when

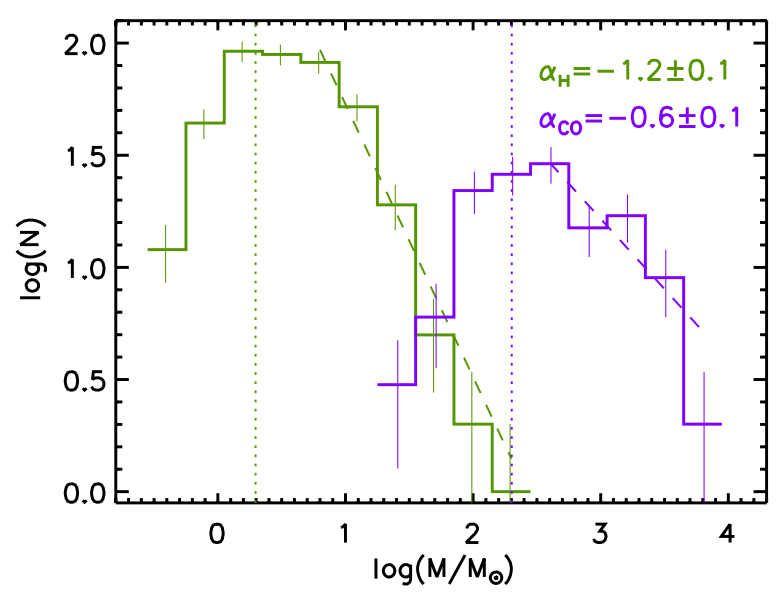

Figure 15. Source mass distribution in $\ell 217-224$ (green: Hi-GAL pre-stellar sources; purple: CO clumps). The error bars are estimated as $\sqrt{N}$ statistical uncertainties. The dashed lines represent the maximum likelihood fit (see Olmi et al. 2013) to the linear portion of the two mass distributions, and the corresponding slopes are reported. As a consequence of the adopted fitting technique, the slope does not depend on the arbitrary choice of the bin width. The dotted vertical lines represent the mass completeness limit in the two cases: for the Hi-GAL observations it has been derived as described in the text, whereas for the NANTEN observations we followed the method of Simon et al. (2001).

(A color version of this figure is available in the online journal.)

the considered sample is contaminated by the presence of larger structures (clumps), as in our case (see Figure 14), the slope generally tends to be shallower, toward the values found for the clump mass functions (e.g., $\alpha=-(0.6-0.8)$ for various clouds, Kramer et al. $1998 ; \alpha=-0.6$ for the Galactic center region, Miyazaki \& Tsuboi 2000; $\alpha=-1.0$ for the Vela-D cloud, Elia et al. 2007). A very similar behavior was also observed in the case of the Herschel-based source mass distribution of the Vela-C cloud ( $\alpha=-1.1 \pm 0.2$; Giannini et al. 2012) affected by the same kind of contamination.

In our case, we can check on the same region this gradual steepening of the mass function from clumps to cores, by considering the masses of the CO clumps extracted as described in Section 4.2, and belonging to the component I (purple histogram in Figure $15, \alpha=-0.7 \pm 0.3$ ). This steepening is quite usual and is explained, in the general case, as a consequence of the fragmentation of the clumps in cores that constitute the last fragment generating the single star (or multiple system). However, in our case, notice that the error bars associated with the two slopes (obtained with the same method) partially overlap. Furthermore, the disagreement between Herschel- and CO-derived column densities highlighted in Figure 2 surely introduces an uncertainty on the slope of the CO clump mass function larger than that derived simply from the power-law fit.

It is possible to make a direct comparison with the mass function slope derived by Kim et al. (2004) for their "Group I" clumps (namely, sources associated with CMa OB1 and G220.8-1.7), $\alpha=-0.59 \pm 0.32$, which is consistent with our result.

\section{EVOLUTIONARY FRAMEWORK}

To further investigate the differences between the proto- and pre-stellar sources considered so far, we discuss here a plot of the bolometric luminosities versus the envelope masses. In the previous literature (Saraceno et al. 1996; André et al. 2000; Molinari et al. 2008) such a diagram has already been used as a meaningful method of inferring the evolutionary status of the 
compact sources, through a comparison of their positions in the plot and the theoretical tracks obtained from models of accreting cores. Such tracks start from a given initial mass and initially follow an almost vertical path corresponding to the accretion phase. Subsequently, the proto-stellar outflow activity produces mass loss and disperses the residual envelope (horizontal portion of the tracks).

Recently, these diagnostics have been applied to the clump populations of star-forming regions observed by Herschel (Elia et al. 2010; Bontemps et al. 2010; Hennemann et al. 2010; Giannini et al. 2012; Veneziani et al. 2013), revealing some degree of segregation between proto- and pre-stellar sources. A general caveat about the use of this diagram for distant starforming regions consists of the fact that, rigorously speaking, the evolutionary tracks are calculated for a single proto-star. Instead, in cases like ours, many sources are in fact clumps having an unresolved internal structure, with the possible presence of a few proto-stars, and/or smaller pre-stellar cores. Therefore, a precise star formation timeline cannot be deduced from such tracks. Of course, in these cases, to find a higher luminosity at the same envelope mass is indicative of a more evolved evolutionary stage, but it is difficult to understand whether this is a general property of the entire (unresolved) population hosted by the clump, or essentially due to only the brightest contained source(s).

To build this diagram, we used the mass values discussed in the previous sections. They represent, in fact, the envelope mass in the case of a proto-stellar source, and of course the whole mass of a starless one. The luminosities have been derived by integrating the whole observed SED, including (1) the flux of the best-fitting modified blackbody at $1 \mathrm{~mm}$, to also take into account the millimetric portion of the SED we do not observe directly, and (2) the WISE flux at $22 \mu \mathrm{m}$, where available, to ensure a better estimate in the case of the sources most evolved and then bright in the mid-infrared unlike, for example, the similar plot in Giannini et al. (2012). By the way, in Molinari et al. (2008) and Hennemann et al. (2010), since their targets were constituted by limited amounts of proto-stellar sources (this explains the lack of the pre-stellar ones in their analysis), more extended SEDs were collected, down to 8.3 and $3.6 \mu \mathrm{m}$, respectively. On the one hand, this increases and improves the bolometric luminosity estimation for more evolved sources; on the other hand, this operation requires special care in checking the reliability of the short-wavelength counterpart assignment to each single FIR source, and therefore it lies outside the goals of the present work.

The $L_{\text {bol }}$ versus $M_{\text {env }}$ plot is shown in Figure 16, with the same symbol convention as that in Figure 14. Furthermore, the symbol size linearly scales with the source temperature. The theoretical evolutionary tracks for the low- and high-mass regimes adopted by Molinari et al. (2008) are also plotted.

Also in our case, the proto-stellar sources and the pre-stellar ones populate quite different regions of the diagram, corresponding to the accreting core phase in the first case, and to a quiescent or collapsing core in the second, respectively. Obviously, there is a transition region with some overlap between the two populations, especially at high masses (discussed below), but a global look suggests a certain degree of segregation.

In the low-mass regime, where the distinction of well-defined evolutionary classes makes more sense (André et al. 2000, and references therein), the region corresponding to the Class 0 to I transition appears populated, as in Giannini et al. (2012). However, we remind the reader that the $L_{\text {bol }}$ versus $M_{\text {env }}$ plot

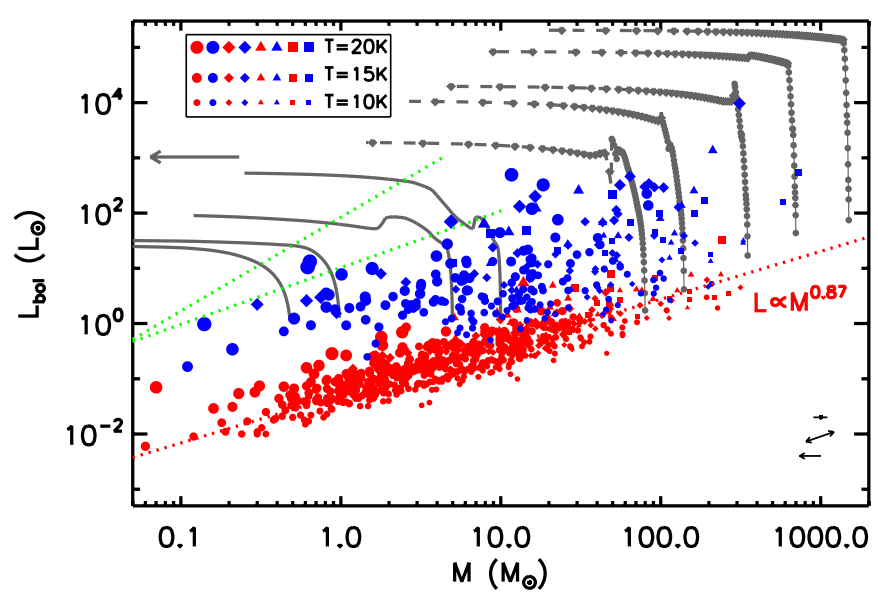

Figure 16. Plot of the bolometric luminosity vs. the mass of the same sources of Figure 14, with the same symbol and color convention. In addition, the symbol size varies according to the source temperature, as illustrated in the legend. Gray and black solid lines represent the evolutionary tracks for the low- and high-mass regimes, respectively, taken from Molinari et al. (2008). The gray arrow indicates the evolution direction, while dashed green lines delimit the region of transition between Class 0 and Class I sources (André et al. 2000) in the low-mass regime. The red dotted line represents the best-fitting power law $\left(L_{\mathrm{bol}} \propto M_{\mathrm{env}}^{0.87 \pm 0.02}\right.$ ) for the distribution of the pre-stellar sources. In the bottom right corner the effects of possible sources of random or systematic uncertainty affecting the points of the plot are represented as arrows, as in Figure 14 (see that figure for further explanation).

(A color version of this figure is available in the online journal.)

is not a tool suitable for obtaining a precise classification, essentially because (1) Hennemann et al. (2010) showed that sources classified as Class I from their NIR-to-MIR SED are found to be compatible instead with a Class 0 status based on their FIR fluxes, and there is not a sharp transition from Class 0 to I to II in their $L_{\text {bol }}$ versus $M_{\text {env }}$ diagram and (2) this evolutionary classification cannot be easily extended to the highmass regime, a fortiori when the considered source is a large clump hosting multiple star formation. Furthermore, comparing our diagram with those of Molinari et al. (2008) and Hennemann et al. (2010), we can explain the lack of evolved sources in ours with the aforementioned different ranges used for calculating $L_{\text {bol }}$. In summary, due to both the investigated spectral range and mass regime, this $L_{\text {bol }}$ versus $M_{\text {env }}$ plot cannot be used to obtain a classical single-YSO classification.

From the point of view of the distance, it is remarkable that proto-stellar activity is also detected in the most distant sources, also confirming the occurrence of the star formation at $R>11$ kpc (see, e.g., Reipurth \& Yan 2008; Yun et al. 2009, and references therein).

Focusing on the source temperatures, the first general indication is that the most luminous proto-stellar sources, i.e., the most evolved, are also the warmest, and of course the protostellar sources are on average warmer than the pre-stellar ones as already indicated by Figure 12. Furthermore, the luminous pre-stellar sources occupying regions of the diagram where early-stage proto-stellar cores are instead expected (beginning of the evolutionary tracks) are generally characterized by a higher temperature. We interpret this fact as the presence of a strongly embedded early-phase proto-star(s) not detected at either the 22 or $70 \mu \mathrm{m}$ wavebands. This might lead to misclassifying early proto-stellar sources as pre-stellar, a fact that should be taken into account in the future analyses of core/clump populations extracted from the Herschel maps. 
Finally, we notice that while the proto-stellar sources are spread over a wide range of possible evolutionary stages, the displacement of the bulk of the pre-stellar sources follows a power law in a region of the diagram corresponding to a stage in which no mass accretion has started yet. A similar behavior was already discussed in Giannini et al. (2012) for the Vela-C cloud but here it looks more evident and, more interestingly, it appears the same over several spatially unrelated regions. A power-law fit gives a dependence $L_{\text {bol }} \propto M^{(0.87 \pm 0.02)}$. This slope is very similar to the 0.85 value found by Brand et al. (2001) for their sample of proto-stellar sources, while it is very different from the 0.42 value found by Giannini et al. (2012) for pre-stellar sources in Vela-C. However, we need to increase the statistics of this slope across the Galactic plane, exploiting the whole Hi-GAL database, before stating a possible link between the evolutionary stage of a star-forming region and the slope of the luminosity versus mass relation of the pre-stellar cores.

\subsection{Star Formation Rate}

The $L_{\text {bol }}$ versus $M_{\text {env }}$ diagram can be exploited to quantify the star formation rate (SFR) of the four distance components we identify in $\ell 217-224$. For the high-mass regime $\left(M>10 M_{\odot}\right)$, we followed the method of Veneziani et al. (2013). Briefly, it consists of associating each proto-stellar source mass with a nearby evolutionary track, and to consider the mass $M_{f}$ of the central object at the end of the track (corresponding to a time $t_{f}$ ), when the envelope clean-up phase is completed. The SFR is calculated by adding the $M_{f} / t_{f}$ ratios of all sources. In the low-mass regime, instead, we use Equation (1) of Lada et al. (2010) which assumes a constant final mass and time, $0.5 M_{\odot}$ and $2 \times 10^{6} \mathrm{yr}$, respectively. The contribution of the low-mass part to the total SFR amounts to $8 \%$ for component I and less than $1 \%$ for the remaining components. We find, for the components I-IV, SFR $=2.6_{-0.3}^{+0.2} \times 10^{-4}, 1.39_{-0.05}^{+0.03} \times$ $10^{-4}, 6.30_{-0.23}^{+0.04} \times 10^{-5}$, and $4.77_{-0.05}^{+0.26} \times 10^{-5} M_{\odot} \mathrm{yr}^{-1}$, respectively. In the portion of the Galaxy corresponding to $\ell 217-224$, thus, $\mathrm{SFR}_{\text {tot }}=5.1_{-0.3}^{+0.2} \times 10^{-4} M_{\odot} \mathrm{yr}^{-1}$. The error bars have been estimated assuming a $20 \%$ average uncertainty on both mass and luminosity. Of course, larger uncertainties come from the choice of the YSO model and from the distance estimate and are not easily quantifiable (see Veneziani et al. 2013).

In two single Hi-GAL tiles of the first Galactic quadrant, namely centered close to $[\ell, b]=\left[30^{\circ}, 0^{\circ}\right]$ and $\left[59^{\circ}, 0^{\circ}\right]$, Veneziani et al. (2013) obtained SFR $=9.5 \times 10^{-4}$ and $1.6 \times 10^{-4} M_{\odot} \mathrm{yr}^{-1}$, respectively, considering all the distance components together. Taking into account that the solid angle spanned by the $\ell 217-224$ maps is about five times larger than in the case of these two inner Galaxy fields, we conclude that in our case the SFR per solid angle is significantly lower, as expected for the outer Galaxy. However, forthcoming studies based on the Hi-GAL survey will provide a more exhaustive description of the SFR as a function of the Galactic position, and an extrapolation to the SFR of the entire Galaxy.

\subsection{Diagnostics of CO Clumps}

If one wants to apply diagnostics based on a luminosity-mass relation to the clumps extracted from the $\mathrm{CO}(1-0)$ data, the most meaningful way to do so is to use the virial mass. Indeed, the way the clump mass is calculated in our case, namely as proportional to the intensity integrated over all the channels assigned by the cloud decomposing algorithm

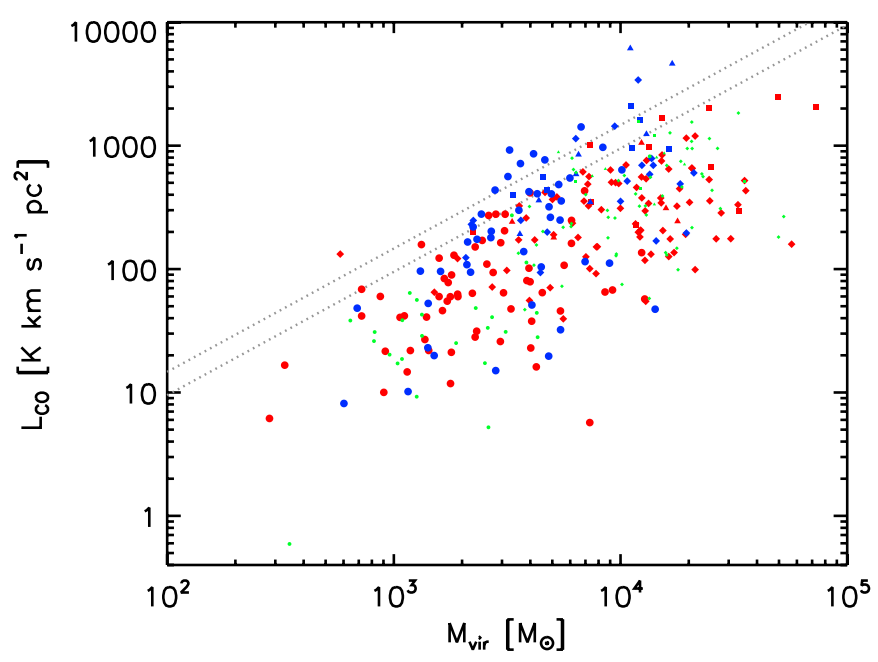

Figure 17. Plot of the luminosity vs. the virial mass of the CO clumps, derived as in Section 4.2. The symbol-distance convention is the same as that in Figure 14. The blue color indicates clumps containing proto-stellar Hi-GAL sources (at least one), while the red color indicates clumps associated only with starless HiGAL sources. Sources lacking Hi-GAL counterparts or lying outside the area surveyed by Herschel are plotted with small green symbols. Two gray dotted lines represent the luminosity vs. luminous mass relation for the two extreme values of the $X$ factor used in this paper.

(A color version of this figure is available in the online journal.)

to the clump (Equation (3)), makes it directly proportional to the luminosity, which, strictly speaking, is a "luminous mass":

$$
M_{\mathrm{CO}}=c L_{\mathrm{CO}}
$$

Bolatto et al. (2008), who used the same decomposition algorithm we used, report a value for the constant $c=45$ with $M_{\mathrm{CO}}$ in $M_{\odot}$ and $L_{\mathrm{CO}}$ in $\mathrm{K} \mathrm{km} \mathrm{s}^{-1} \mathrm{pc}^{2}$. On the contrary, the estimate of the virial mass is independent of the clump luminosity. We show the plot of these two quantities (derived as in Section 4.2) in Figure 17(a), where we adopt the same symbol convention as in Figure 16 to separate sources associated with the different distance components. Furthermore, blue and red colors are used for clumps associated with at least one Hi-GAL proto-stellar source and clumps associated only with Hi-GAL starless ones, respectively. Finally, smaller green symbols represent the $\mathrm{CO}$ clumps that do not contain Hi-GAL sources or that lie outside the area surveyed by Herschel.

Two lines corresponding to Equation (7) for two different slopes have been plotted (gray dotted lines). They are obtained correcting the $c$ value of Bolatto et al. (2008, corresponding to $\left.X=2 \times 10^{20}\left(\mathrm{~K} \mathrm{~km} \mathrm{~s}^{-1}\right)^{-1} \mathrm{~cm}^{-2}\right)$, by the minimum (upper line) and maximum (lower line) values of $X$ used for the clumps of this paper, respectively, according to Equation (3). This is equivalent to saying that all the values of the luminosity-luminous mass pairs lie in the stripe of the plot delimited by these two lines (and in particular the majority of them lie close to the upper one, namely that corresponding to the smaller value of $X$ ).

The less rigorous classification between "proto-stellar" and "starless" CO clumps probably explains the reason for the absence of a clear segregation like that found in Figure 16 for dust cores and clumps. It is noteworthy, however, that for $M_{\mathrm{CO}} \gg 10^{3} M_{\odot}$ the most luminous clumps (1) all have a proto-stellar content and (2) all are supervirial. If we consider all the supervirial clumps in our sample, namely 22 objects corresponding to $\sim 7 \%$ of the total, 17 of them have a protostellar content, 3 are starless, and 2 are not associated with 
Herschel sources. This indication is surely relevant, but not conclusive or sufficient enough to identify evolved clumps in such a diagram. On the one hand, the majority of the supervirial structures are found to host star formation; on the other hand, many "proto-stellar" clumps can appear as sub-virial since their global physical properties plotted here cannot describe local conditions that could enable star formation in limited parts of them. Finally, unlike the case of the Hi-GAL $L_{\text {bol }}$ versus $M$ diagram, we do not recognize any power-law trend in either the whole sample of clumps or the single identified classes.

\section{CONCLUSIONS}

The first Hi-GAL observations of the TGQ consist of a "chunk" of $\sim 20.5 \mathrm{deg}^{2}$ corresponding to the longitude range $216.5 \leqslant \ell \leqslant 225.5$. Here we presented a first study based on both the overall displacement of the far-infrared emission in this region and on the statistical analysis of the properties of the compact sources derived from photometric measurements. The NANTEN CO(1-0) observations have added fundamental information about gas kinematics. This study confirms the view of the outer Galaxy as an interesting laboratory in which to investigate star formation processes, benefiting from the generally simple structure of the velocity field (compared with the inner Galaxy). Summarizing, we found the following.

1. In the Hi-GAL images, it is possible to recognize the dust emission from all the velocity components in this sky region detected in the line observations presented in this and previous papers (Kim et al. 2004). In particular, we identify four components (I-IV) located at average heliocentric distances of 1.1,2.2,3.3, and $5.8 \mathrm{kpc}$, respectively. The overlap among these components is quite infrequent, making their identification clearer.

2. The large-scale column density and temperature distribution for the cold component of the dust reveal a variety of conditions, with star formation sites preferentially distributed along filaments. Interestingly, two of these are likely associated with the S296 H II region, which might be responsible for triggering star formation in these filaments.

3. We obtained a five-band Hi-GAL compact source catalog of this region, complemented by the WISE $22 \mu \mathrm{m}$ photometry and the distance information derived from the spatial association with the clumps extracted from the $\mathrm{CO}(1-0)$ data set. The classification between proto-stellar (provided with a detection at $70 \mu \mathrm{m}$ and/or at $22 \mu \mathrm{m}$ and $160 \mu \mathrm{m}$ ) and starless sources has been made.

4. A modified blackbody has been fitted to the regular and well-sampled SEDs (255 proto-stellar; 688 starless) to derive temperatures and masses. On average, the protostellar sources are found to be warmer than the starless ones. Moreover, concerning the source size, we find that our sample is composed of a mixture of clumps and cores, due to the wide involved distance range. Looking at their spatial distribution in the Galactic plane, the local and Perseus spiral arms are clearly seen, although a remarkable amount of sources with assigned inter-arm distances are also found. The limited range of investigated Galactic longitudes does not afford a better recognition of a grand design spiral pattern.

5. We derived the SFE for the four velocity components $(0.008,0.004,0.012$, and 0.006 , respectively). These values lead to classifying the star formation in these regions as not very efficient. On the one hand, being based only on Herschel far-infrared photometry, they should be taken as lower limits. On the other hand, a systematic overestimate of the SFE with increasing distance has to be taken into account.

6. Applying a virial analysis to the starless Hi-GAL sources, large fractions of them (ranging from $82 \%$ for component I to $100 \%$ for component IV) are found to be gravitationally bound, i.e., pre-stellar. We show that these percentages must be considered as upper limits because they are biased by a selection effect related to the distance and implicit in the adopted criterion.

7. In the mass versus radius plot for the pre-stellar sources we do not recognize any clear power-law behavior. We use this tool to check the potential ability of clumps/cores to form massive stars, based on the different thresholds of Krumholz \& McKee (2008) and Kauffmann \& Pillai (2010). In both cases, we find condensations compatible with high-mass star formation in all the distance components.

8. A power law $N(\log M) \propto M^{\alpha}$ has been fitted to the highmass portion of the core/clump mass distribution of the pre-stellar sources belonging to component I. Similar to the Vela-C case (Giannini et al. 2012), its slope $\alpha=-1.0 \pm 0.2$ is shallower (although still consistent within the errors) than the stellar initial mass function slope and the similar slopes also found for cores in nearby star-forming regions, and steeper than the slopes found for the gas clump distributions, as in this case $(\alpha=-0.7 \pm 0.3)$.

9. The $L_{\text {bol }}$ versus $M_{\text {env }}$ plot of the Hi-GAL sources reveals an evident separation between proto- and pre-stellar sources. For both classes the warmest sources are, in general, the most evolved of their class. Few proto-stellar sources populate the region of the diagram where objects in transition between Class 0 and Class I are expected to lie, whereas the majority populate the Class 0 region. This classification obviously weakens when distant sources are considered, namely those corresponding to large and massive $\left(M \gtrsim 100 M_{\odot}\right)$ clumps. Using this diagram as in Veneziani et al. (2013) we are also able to derive an estimate of the star formation rate of $\mathrm{SFR}_{\mathrm{tot}}=5.1 \times 10^{-4} M_{\odot} \mathrm{yr}^{-1}$.

10. Most of the CO clumps $(93 \%)$ are sub-virial. Line observations at better angular resolution would probably reveal denser supervirial substructures. The remaining $7 \%$ of supervirial clumps (22 objects) is in most cases associated with Hi-GAL proto-stellar sources, suggesting that these clumps globally and not just locally fulfill the conditions for the gravitational collapse.

Besides the characterization of star-forming regions in the $\ell 217-224$, this paper also gives a first contribution to the larger statistics that will be built exploiting the entire Hi-GAL survey archive. Prescriptions and caveats for using the photometric data, for combining them with kinematics derived from $\mathrm{CO}$ observations, and for addressing the effects of distance resolution have been explicitly discussed in several points of the text.

PACS has been developed by a consortium of institutes led by MPE (Germany) and including UVIE (Austria); KU Leuven, CSL, IMEC (Belgium); CEA, LAM (France); MPIA (Germany); INAF-IFSI/OAA/OAP/OAT, LENS, SISSA (Italy); and IAC (Spain). This development has been supported by the funding agencies BMVIT (Austria), ESAPRODEX 

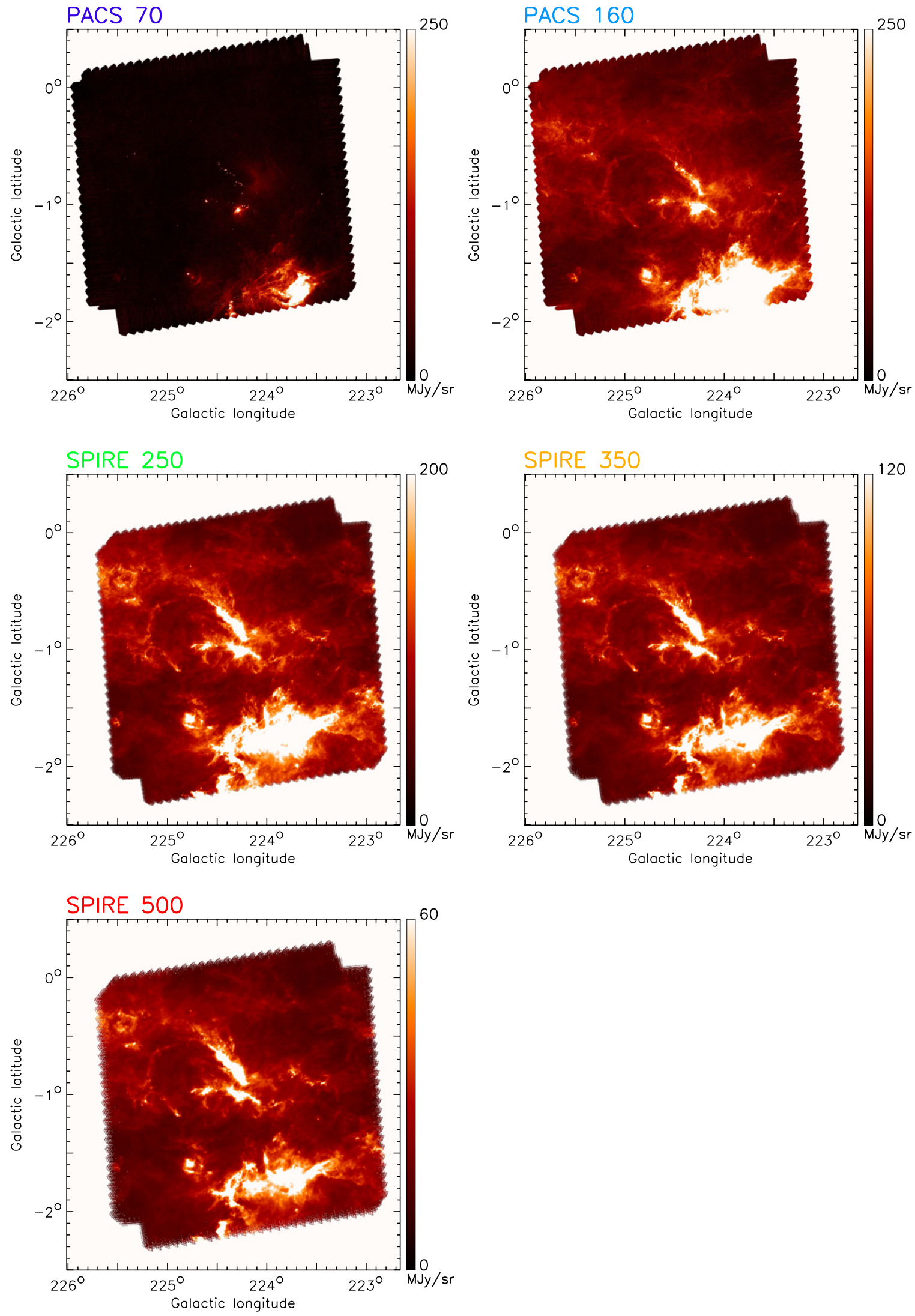

Figure 18. Hi-GAL maps of the $\ell 224$ field at 70, 160, 250, 350, and $500 \mu \mathrm{m}$.

(An extended, color version of this figure is available in the online journal.) 
Table 2

List of the Clumps Detected in the $\mathrm{CO}(1-0)$ Line

\begin{tabular}{lccccccccc}
\hline \hline ID & $\begin{array}{c}\ell \\
\left({ }^{\circ}\right)\end{array}$ & $\begin{array}{c}b \\
\left({ }^{\circ}\right)\end{array}$ & $\begin{array}{c}v_{\text {lsr }} \\
\left(\mathrm{km} \mathrm{s}^{-1}\right)\end{array}$ & $\begin{array}{c}\sigma_{v} \\
\left(\mathrm{~km} \mathrm{~s}^{-1}\right)\end{array}$ & $\begin{array}{c}d \\
(\mathrm{kpc})\end{array}$ & $\begin{array}{c}r \\
(\mathrm{pc})\end{array}$ & $\begin{array}{c}M \\
\left(M_{\odot}\right)\end{array}$ & \multicolumn{1}{c}{$\begin{array}{c}M_{\text {vir }} \\
\left(M_{\odot}\right)\end{array}$} & $\begin{array}{c}L \\
\left(\mathrm{~K} \mathrm{~km} \mathrm{~s}^{-1} \mathrm{pc}^{2}\right)\end{array}$ \\
\hline 1 & 216.61829 & -1.82868 & 44.0 & 1.8 & 4.3 & 5.7 & 5770.0 & 19154.0 & 704.6 \\
2 & 216.62572 & -1.64908 & 30.6 & 0.9 & 2.6 & 6.5 & 2707.0 & 5009.0 & 366.3 \\
3 & 216.64683 & -2.24570 & 23.8 & 1.9 & 1.9 & 3.6 & 3943.0 & 13551.0 & 554.5 \\
4 & 216.66404 & -2.08390 & 25.9 & 2.0 & 2.1 & 5.0 & 6811.0 & 20697.0 & 947.4 \\
5 & 216.66706 & -1.70928 & 32.8 & 0.8 & 2.8 & 8.9 & 5936.0 & 6110.0 & 791.9 \\
6 & 216.67371 & -1.42988 & 30.9 & 1.1 & 2.6 & 5.5 & 3611.0 & 7170.0 & 487.8 \\
7 & 216.68618 & -0.48407 & 55.2 & 1.7 & 6.1 & 8.7 & 6204.0 & 25071.0 & 671.9 \\
8 & 216.69400 & -1.88198 & 29.7 & 1.6 & 2.5 & 7.8 & 8458.0 & 19509.0 & 1151.3 \\
9 & 216.70952 & -0.44180 & 26.5 & 1.2 & 2.1 & 2.8 & 401.0 & 3964.0 & 55.6 \\
10 & 216.72395 & -2.36709 & 24.1 & 1.5 & 1.9 & 4.0 & 3712.0 & 9794.0 & 521.4 \\
\hline
\end{tabular}

(This table is available in its entirety in a machine-readable form in the online journal. A portion is shown here for guidance regarding its form and content.)

Table 3

List of the Analyzed Hi-GAL Sources

\begin{tabular}{|c|c|c|c|c|c|c|c|c|c|c|c|c|c|c|}
\hline ID & $\begin{array}{l}\ell \\
\left({ }^{\circ}\right)\end{array}$ & $\begin{array}{l}b \\
\left({ }^{\circ}\right)\end{array}$ & $\begin{array}{c}d \\
(\mathrm{kpc})\end{array}$ & $\begin{array}{c}F_{70} \pm \Delta F_{70} \\
(\mathrm{Jy})\end{array}$ & $\begin{array}{c}F_{160} \pm \Delta F_{160} \\
(\mathrm{Jy})\end{array}$ & $\begin{array}{c}F_{250} \pm \Delta F_{250} \\
(\mathrm{Jy})\end{array}$ & $\begin{array}{c}F_{350} \pm \Delta F_{350} \\
(\mathrm{Jy})\end{array}$ & $\begin{array}{c}F_{500} \pm \Delta F_{500} \\
(\mathrm{Jy})\end{array}$ & $\begin{array}{c}r \\
\left({ }^{\prime \prime}\right)\end{array}$ & $\begin{array}{c}M \\
\left(M_{\odot}\right)\end{array}$ & $\begin{array}{c}T \\
(\mathrm{~K})\end{array}$ & $\begin{array}{l}F_{22} \\
(\mathrm{Jy})\end{array}$ & $\begin{array}{l}L_{\text {bol }} \\
\left(L_{\odot}\right)\end{array}$ & $\begin{array}{c}\text { Unbound/Pre-/ } \\
\text { Proto-stellar 0/1/2 }\end{array}$ \\
\hline 1 & 216.66360 & -1.66179 & 2.6 & $\ldots$ & $\ldots$ & $0.43 \pm 0.04$ & $0.28 \pm 0.02$ & $0.17 \pm 0.03$ & 21.0 & 6.4 & 11.9 & $\ldots$ & 0.4 & 1 \\
\hline 2 & 216.68802 & -1.42173 & 2.6 & $\ldots$ & $\ldots$ & $0.29 \pm 0.07$ & $0.29 \pm 0.01$ & $0.12 \pm 0.02$ & 13.6 & 5.9 & 11.5 & $\ldots$ & 0.3 & 1 \\
\hline 4 & 216.69757 & -1.41449 & 2.6 & $\ldots$ & $\ldots$ & $0.89 \pm 0.07$ & $0.79 \pm 0.01$ & $0.51 \pm 0.05$ & 31.0 & 33.4 & 10.1 & $\ldots$ & 1.1 & 1 \\
\hline 5 & 216.74443 & -0.39604 & 2.1 & $\ldots$ & $\ldots$ & $0.46 \pm 0.11$ & $0.52 \pm 0.01$ & $0.27 \pm 0.04$ & 25.6 & 13.4 & 9.9 & $\ldots$ & 0.4 & 1 \\
\hline 6 & 216.78496 & -1.06081 & 2.6 & $0.87 \pm 0.03$ & $2.45 \pm 0.12$ & $3.99 \pm 0.15$ & $2.61 \pm 0.02$ & $1.36 \pm 0.01$ & 16.2 & 53.1 & 12.1 & 0.01 & 17.2 & 2 \\
\hline 9 & 216.82965 & -1.30140 & 2.7 & $\ldots$ & $\ldots$ & $0.62 \pm 0.05$ & $0.39 \pm 0.01$ & $0.22 \pm 0.02$ & 17.7 & 8.0 & 12.6 & $\ldots$ & 0.6 & 1 \\
\hline 10 & 216.84781 & -1.32426 & 2.7 & $\ldots$ & $\ldots$ & $0.83 \pm 0.10$ & $0.66 \pm 0.02$ & $0.36 \pm 0.03$ & 23.0 & 18.8 & 11.3 & $\ldots$ & 1.0 & 1 \\
\hline
\end{tabular}

(This table is available in its entirety in a machine-readable form in the online journal. A portion is shown here for guidance regarding its form and content.)

(Belgium), CEA/CNES (France), DLR (Germany), ASI/INAF (Italy), and CICYT/MCYT (Spain). SPIRE has been developed by a consortium of institutes led by Cardiff Univ. (UK) and including Univ. Lethbridge (Canada); NAOC (China); CEA, LAM (France); INAF-IFSI, Univ. Padua (Italy); IAC (Spain); Stockholm Observatory (Sweden); Imperial College London, RAL, UCL-MSSL, UKATC, Univ. Sussex (UK); and Caltech, JPL, NHSC, Univ. Colorado (USA). This development has been supported by the national funding agencies CSA (Canada); NAOC (China); CEA, CNES, CNRS (France); ASI (Italy); MCINN (Spain); SNSB (Sweden); STFC, UKSA (UK); and NASA (USA). D.E., M.P., and K.J.L.R. are funded by an ASI fellowship under contract numbers I/005/11/0 and I/038/08/ 0. D.P. is funded through the Operational Program "Education and Lifelong Learning" and is co-financed by the European Union (European Social Fund) and Greek national funds.

Facilities: Herschel

\section{APPENDIX A}

Hi-GAL IMAGES: THE $\ell 224, \ell 222, \ell 220$, AND $\ell 217$ TILES FROM 70 TO $500 \mu \mathrm{m}$

Here we present the Hi-GAL basic data used in this article, namely all the single maps obtained for the field $\ell 224, \ell 222$, $\ell 220$, and $\ell 217$ at $160,250,350$, and $500 \mu \mathrm{m}$.

\section{APPENDIX B}

\section{LIST OF CO CLUMPS}

In this appendix the list of the clumps obtained from the NANTEN data cube decomposition and their basic properties (see Section 4.2) is reported.

\section{APPENDIX C}

\section{LIST OF HERSCHEL SOURCES}

In this appendix the list of the compact sources extracted with CuTEx from the Hi-GAL maps (see Section 4.1) and eligible for the modified blackbody fit (see Section 5.1) and their basic properties (see Sections 5.2 and 5.3) is reported.

\section{REFERENCES}

Alves, J., Lombardi, M., \& Lada, C. J. 2007, A\&A, 462, L17

André, P., Men'shchikov, A., Bontemps, S., et al. 2010, A\&A, 518, L102 André, P., Ward-Thompson, D., \& Barsony, M. 2000, in Protostars and Planets IV, ed. V. Mannings, A. P. Boss, \& S. S. Russell (Tucson, AZ: Univ. Arizona Press), 59

Bergin, E. A., \& Tafalla, M. 2007, ARA\&A, 45, 339

Bernard, J.-P., Paradis, D., Marshall, D. J., et al. 2010, A\&A, 518, L88

Blitz, L., Fich, M., \& Stark, A. A. 1982, ApJS, 49, 183

Bolatto, A. D., Leroy, A. K., Rosolowsky, E., Walter, F., \& Blitz, L. 2008, ApJ, 686, 948

Bontemps, S., André, P., Könyves, V., et al. 2010, A\&A, 518, L85

Brand, J., Cesaroni, R., Palla, F. \& Molinari, S. 2001, A\&A, 370, 230 
Brunt, C. M., Kerton, C. R., \& Pomerleau, C. 2003, ApJS, 144, 47

Brunthaler, A., Reid, M. J., Menten, K. M., et al. 2011, AN, 332, 461

Burton, W. B., \& te Lintel Hekkert, P. 1986, A\&AS, 65, 427

Carlhoff, P., Nguyen Luong, Q., Schilke, P., et al. 2013, A\&A, submitted

Carraro, G., Vázquez, R. A., Moitinho, A., \& Baume, G. 2005, ApJL, 630, L153

Chabrier, G. 2005, in The Initial Mass Function 50 Years Later, ed. E. Corbelli, F. Palla, \& H. Zinnecker (Astrophysics and Space Science Library, Vol. 327; Dordrecht: Springer), 41

Clariá, J. J. 1974, A\&A, 37, 229

Cordes, J. M., \& Lazio, T. J. W. 2002, arXiv:astro-ph/0207156

Dame, T. M., Hartmann, D., \& Thaddeus, P. 2001, ApJ, 547, 792

Dobashi, K., Uehara, H., Kandori, R., et al. 2005, PASJ, 57, 1

Egan, M. P., Shipman, R. F., Price, S. D., et al. 1998, ApJL, 494, L199

Elia, D., Massi, F., Strafella, F., et al. 2007, ApJ, 655, 316

Elia, D., Schisano, E., Molinari, S., et al. 2010, A\&A, 518, L97

Enoch, M. L., Evans, N. J., II, Sargent, A. I., et al. 2008, ApJ, 684, 1240

Federrath, C., Glover, S. C. O., Klessen, R. S., \& Schmidt, W. 2008, PhST, 132, 014025

Giannini, T., Elia, D., Lorenzetti, D., et al. 2012, A\&A, 539, A156

Gregorio-Hetem, J., Montmerle, T., Rodrigues, C. V., et al. 2009, A\&A, 506, 711

Griffin, M. J., Abergel, A., Abreu, A., et al. 2010, A\&A, 518, L3

Hennemann, M., Motte, F., Bontemps, S., et al. 2010, A\&A, 518, L84

Heyer, M. H., Brunt, C., Snell, R. L., et al. 1998, ApJS, 115, 241

Heyer, M. H., Williams, J. P., \& Brunt, C. M. 2006, ApJ, 643, 956

Hildebrand, R. H. 1983, QJRAS, 24, 267

Hill, T., Motte, F., Didelon, P., et al. 2011, A\&A, 533, A94

Ikeda, N., Sunada, K., \& Kitamura, Y. 2007, ApJ, 665, 1194

Kainulainen, J., Beuther, H., Banerjee, R., Federrath, C., \& Henning, T. 2011, A\&A, 530, A64

Kauffmann, J., \& Pillai, T. 2010, ApJL, 723, L7

Kim, B. G., Kawamura, A., Yonekura, Y., \& Fukui, Y. 2004, PASJ, 56, 313

Klessen, R. S. 2000, ApJ, 535, 869

Könyves, V., André, P., Men'shchikov, A., et al. 2010, A\&A, 518, L106

Kramer, C., Stutzki, J., Rohrig, R., \& Corneliussen, U. 1998, A\&A, 329, 249

Kroupa, P. 2001, MNRAS, 322, 231

Krumholz, M. R., \& McKee, C. F. 2008, Natur, 451, 1082

Kutner, M. L., \& Ulich, B. L. 1981, ApJ, 250, 341

Lada, C. J., Lombardi, M., \& Alves, J. F. 2010, ApJ, 724, 687

Larson, R. B. 1981, MNRAS, 194, 809

Lee, Y., Snell, R. L., \& Dickman, R. L. 1994, ApJ, 432, 167

Lynds, B. T. 1962, ApJS, 7, 1

Maddalena, R. J., Morris, M., Moscowitz, J., \& Thaddeus, P. 1986, ApJ, 303,375

May, J., Alvarez, H., \& Bronfman, L. 1997, A\&A, 327, 325

May, J., Bronfman, L., Alvarez, H., Murphy, D. C., \& Thaddeus, P. 1993, A\&AS, 99, 105

May, J., Murphy, D. C., \& Thaddeus, P. 1988, A\&AS, 73, 51

Megeath, S. T., Allgaier, E., Young, E., et al. 2009, AJ, 137, 4072

Miyazaki, A., \& Tsuboi, M. 2000, ApJ, 536, 357

Mizuno, A., \& Fukui, Y. 2004, in ASP Conf. Ser. 317, Milky Way Surveys:

The Structure and Evolution of Our Galaxy, ed. D. Clemens, R. Shah, \& T. Brainerd (San Francisco, CA: ASP), 59
Moitinho, A., Vázquez, R. A., Carraro, G., et al. 2006, MNRAS, 368, L77

Molinari, S., Pezzuto, S., Cesaroni, R., et al. 2008, A\&A, 481, 345

Molinari, S., Schisano, E., Faustini, F., et al. 2011, A\&A, 530, A133

Molinari, S., Swinyard, B., Bally, J., et al. 2010a, A\&A, 518, L100

Molinari, S., Swinyard, B., Bally, J., et al. 2010b, PASP, 122, 314

Motte, F., \& André, P. 2001, A\&A, 365, 440

Motte, F., Zavagno, A., Bontemps, S., et al. 2010, A\&A, 518, L77

Murray, N. 2011, ApJ, 729, 133

Nakanishi, H., \& Sofue, Y. 2006, PASJ, 58, 847

Ogawa, H., Mizuno, A., Ishikawa, H., Fukui, Y., \& Hoko, H. 1990, IJIMW, 11,717

Olmi, L., Anglés-Alcázar, D., De Luca, M., et al. 2010, ApJ, 723, 1065

Olmi, L., Anglés-Alcázar, D., Elia, D., et al. 2013, A\&A, 551, A111

Ott, S. 2010, in ASP Conf. Ser. 434, Astronomical Data Analysis Software and Systems XIX, ed. Y. Mizumoto, K.-I. Morita, \& M. Ohishi (San Francisco, CA: ASP), 139

Piazzo, L., Ikhenaode, D., Natoli, P., et al. 2012, ITIP, 21, 3687

Pilbratt, G. L., Riedinger, J. R., Passvogel, T., et al. 2010, A\&A, 518, L1

Pineda, J. E., Caselli, P., \& Goodman, A. A. 2008, ApJ, 679, 481

Poglitsch, A., Waelkens, C., Geis, N., et al. 2010, A\&A, 518, L2

Preibisch, T., Ossenkopf, V., Yorke, H. W., \& Henning, T. 1993, A\&A, 279, 577

Reid, M. J., Menten, K. M., Zheng, X. W., et al. 2009, ApJ, 700, 137

Reipurth, B., \& Yan, C.-H. 2008, in Handbook of Star Forming Regions, Vol. I: The Northern Sky, ed. B. Reipurth (ASP Monograph Publications, Vol. 4; San Francisco, CA: ASP), 869

Rosolowsky, E., \& Blitz, L. 2005, ApJ, 623, 826

Rosolowsky, E., \& Leroy, A. 2006, PASP, 118, 590

Rosolowsky, E., \& Leroy, A. 2011, ascl soft, record ascl:1102.012, 2012

Ruprecht, J. 1966, IAU Trans., XIIB, 348

Russeil, D., Pestalozzi, M., Mottram, J. C., et al. 2011, A\&A, 526, A151

Sadavoy, S. I., di Francesco, J., André, P., et al. 2012, A\&A, 540, A10

Saraceno, P., Andre, P., Ceccarelli, C., Griffin, M., \& Molinari, S. 1996, A\&A, 309,827

Schneider, N., Csengeri, T., Hennemann, M., et al. 2012, A\&A, 540, L11

Sharpless, S. 1959, ApJS, 4, 257

Shetty, R., Glover, S. C., Dullemond, C. P., \& Klessen, R. S. 2011, MNRAS, 412, 1686

Simon, R., Jackson, J. M., Clemens, D. P., Bania, T. M., \& Heyer, M. H. 2001, ApJ, 551, 747

Solomon, P. M., Downes, D., \& Radford, S. J. E. 1992, ApJL, 398, L29

Solomon, P. M., Rivolo, A. R., Barrett, J., \& Yahil, A. 1987, ApJ, 319, 730

Tegmark, M. 1997, ApJL, 480, L87

Traficante, A., Calzoletti, L., Veneziani, M., et al. 2011, MNRAS, 416, 2932

Vallée, J. P. 2005, AJ, 130, 569

Vázquez, R. A., May, J., Carraro, G., et al. 2008, ApJ, 672, 930

Veneziani, M., Elia, D., Noriega-Crespo, A., et al. 2013, A\&A, 549, A130

Williams, J. P., de Geus, E. J., \& Blitz, L. 1994, ApJ, 428, 693

Wouterloot, J. G. A., Brand, J., Burton, W. B., \& Kwee, K. K. 1990, A\&A, 230,21

Wright, E. L., Eisenhardt, P. R. M., Mainzer, A. K., et al. 2010, AJ, 140, 1868

Xu, Y., Voronkov, M. A., Pandian, J. D., et al. 2009, A\&A, 507, 1117

Yun, J. L., Elia, D., Palmeirim, P. M., Gomes, J. I., \& Martins, A. M. 2009, A\&A, 500,833 